\title{
Externalizing the lateral boundary conditions from the dynamic core in semi-implicit semi-Lagrangian models
}

\author{
By PIET TERMONIA ${ }^{1 *}$ and FABRICE VOITUS ${ }^{2},{ }^{1}$ Royal Meteorological Institute, Brussels, Belgium; \\ ${ }^{2}$ Météo-France/CNRM/GMAP, Toulouse, France
}

(Manuscript received 28 August 2007; in final form 14 April 2008)

\begin{abstract}
Research is still undertaken to develop so-called transparent lateral boundary conditions (LBC) for limited-area numerical weather prediction models. In the widely used semi-implicit semi-Lagrangian schemes, this naturally leads to LBC formulations that are intrinsically intertwined with the numerics of the dynamic core. This may have profound consequences for the implementation and the maintenance of future model codes. For instance, scientific development on the dynamics may be hindered by constraints coming from today's choices in the LBC formulation and vice versa.

Building further on the work of Aidan McDonald, this paper proposes an approach where (1) the LBCs can be imposed by an extrinsic numerical scheme that is fundamentally different from the one used for the dynamic core in the interior domain and (2) substituting one such LBC scheme for another is possible in a manner that leaves the Helmholtz solver unmodified. It is argued that this concept may provide the necessary frame for formulating transparent boundary conditions in spectral limited-area models.

Since this idea touches all aspects of the LBC problem, its feasibility can only be established by a rigorous systematic approach. As a first step, this paper provides promising experimental support in a one-dimensional shallow-water model.
\end{abstract}

\section{Introduction}

Notwithstanding the success of the Davies scheme (Davies, 1976) in operational meteorological atmospheric models, active work on the formulation of transparent boundary conditions has still been carried out in recent years. In particular, the work of McDonald (2000, 2003, 2005, 2006) in the HIRLAM model has led to a formulation of quality comparable to the Davies scheme (McDonald, personal communication, 2007).

In such transparent formulations of the lateral-boundary conditions (LBCs), one variable is specified at the boundary for each of the incoming characteristics while the remaining ones are integrated in an upstream manner. The choice of the variables and the way they are imposed are not unique. In fact, different choices lead to different accuracy properties of the resulting scheme, see for instance McDonald (2000).

In practical implementations, such formulations tend to become 'intrinsically' intertwined with the dynamics of the model at the boundaries of the domain. In semi-implicit schemes, the solver of the Helmholtz equation of the dynamic core needs to be adapted accordingly. Moreover, the details of the solver may depend on the chosen LBC implementation. Each new LBC for-

\footnotetext{
*Corresponding author.

e-mail: piet.termonia@oma.be

DOI: $10.1111 /$ j.1600-0870.2008.00334.x
}

mulation a priori necessitates to revisit its implementation. Vice versa, the development of the dynamic core may be constrained by the specificities of the present LBC formulation.

This paper, in the context of the widely used two-time-level semi-implicit semi-Lagrangian (2TL SISL) schemes, (1) investigates whether imposing the LBCs can be done with numerical schemes that are fundamentally different from the one used for the dynamic core and (2) proposes an approach where the integration algorithm of the dynamic core in the interior domain is independent of the details of the chosen LBC scheme. As will be shown, such an 'extrinsic' LBC scheme is a gridpoint scheme in a buffer zone near the boundary.

This concept will be referred to as 'externalizing' the LBCs. Externalizing problems in atmospheric model codes is not new. Indeed, Best et al. (2004) proposed a numerical method to externalize the treatment of the surface from the atmospheric part of the model, facilitating the study of both as distinct scientific entities.

The idea of externalizing the LBCs is a fundamental one, with potentially far reaching consequences on the way LBCs can be managed within atmospheric or oceanic models. However, the answer as to whether this is feasible, requires a rigorous systematic approach where all the different aspects of transparent boundary conditions are controlled. As a first step this paper presents tests in a one-dimensional shallow-water equation, similar to the one used by McDonald (2000). As in that paper, this 
model will be discretized by a 2TL SISL scheme on an Arakawa $\mathrm{C}$ grid and will be coupled to the analytic solution of the equations (instead of a nesting to a host model as in the later paper McDonald, 2003) to eliminate any problems due to incompatibilities between the coupling model and the coupled model of the type mentioned by Warner et al. (1997). Avoiding such subtleties, it should be possible to approximate the analytic solution of the system almost exactly. It will be shown below that for the studied externalized LBC treatment, this is indeed possible, providing substantial evidence for the idea.

The search for transparent boundary conditions in spectral limited-area models (LAMs) poses a particular challenge. It is impossible to adapt gridpoint values locally in spectral space. This is a fortiori a problem for the LBCs on the gridpoints of the boundaries. Although the primary goal of this paper is not to solve this issue, it will be shown that the proposed concept of externalization can be extended to spectral models. This externalization allows, by construction, to impose the LBCs outside the spectral Helmholtz equation, in the extrinsic gridpoint LBC scheme near the boundary, with the same stability properties as the implementations in gridpoint models.

Studying the spectral model alongside the gridpoint model and controlling all its features, allows to identify the extra complications purely caused by the spectral nature of the model. So, even if the only aim would be to investigate the potential of this concept for solving the problems in spectral models, the externalization must be studied in comparison to an implementation in a gridpoint model. Both are for this reason treated together in this paper.

Tests with the shallow-water model will be presented within a spectral model having a spectral structure that imitates, as closely as possible, the features of the existing operational ALADIN model (ALADIN International team, 1997) which follows the proposal of Haugen and Machenhauer (1993). These tests exhibit some accuracy defects, which are not present in gridpoint models but which are, nevertheless, acceptably small. The cause of these defects have been identified. It will be argued that there is substantial room for improvement within the geometrical configuration of the ALADIN model to make this externalization more accurate. The further treatment of these issues, which we deem necessary for an exhaustive assessment of the problems in a spectral model, lies, however, beyond the scope of this paper.

Although the focus in the paper is put on SISL schemes because of their wide use in numerical weather prediction, the general approach proposed here may have an application in other schemes as well.

This paper is organized as follows. Section 2 introduces the one-dimensional shallow-water model. In this section, a clear distinction will be made between an LBC implementation that modifies the Helmholtz equation of a gridpoint model intrinsically and the idea of the externalization. The difference between intrinsic LBCs and a model with externalized LBCs is explained in the Sections 2.1 and 2.2 respectively. Section 2.3 describes the externalization for the spectral version of the model. The details of how the extrinsic LBCs are computed will be treated separately in the next Section 3. Three examples of such integration schemes that have been used to test the feasibility of substituting one for another, will be described. Section 4 provides experimental support for the presented concept. The results will be discussed and an outlook will be given in Section 5 .

\section{The one-dimensional shallow-water model}

The model under consideration in this paper is the linearized shallow-water system for one-dimensional flow on an $f$-plane, given by the equations

$$
\begin{aligned}
& \frac{\mathrm{d} u(x, t)}{\mathrm{d} t}=-\mathrm{e}^{\bar{\Phi} \frac{\partial \Phi(x, t)}{\partial x}}+f v(x, t), \\
& \frac{\mathrm{d} v(x, t)}{\mathrm{d} t}=-f u(x, t), \\
& \frac{\mathrm{d} \Phi(x, t)}{\mathrm{d} t}=-\frac{\partial u(x, t)}{\partial x},
\end{aligned}
$$

with $x$ and $t$ space and time, $u(x, t)$ and $v(x, t)$ are the components of the wind and $\Phi(x, t)=\ln \phi$ the logarithmic geopotential ( $\phi$ being the geopotential). Without loss of generality, $\bar{u} \geq 0$ will be assumed in this paper. The choice of the logarithm of $\phi$ was taken to follow the approach of the ALADIN model. The total derivative is defined as $\mathrm{d} / \mathrm{d} t=\partial / \partial t+\bar{u} \partial / \partial x$. The advection velocity $\bar{u}$, the reference logarithmic geopotential $\bar{\Phi}$ and the Coriolis parameter $f$ are all constant.

The linear system in eqs. (1)-(3) can be rewritten in matrix notation: $\mathrm{d} \mathbf{X} / \mathrm{d} t=\mathbf{L} \mathbf{X}$, with

$$
\mathbf{X}=\left(\begin{array}{c}
u \\
v \\
\Phi
\end{array}\right) \quad \text { and } \quad \mathbf{L}=\left(\begin{array}{ccc}
0 & f & -\bar{c}^{2} \frac{\partial}{\partial x} \\
-f & 0 & 0 \\
-\frac{\partial}{\partial x} & 0 & 0
\end{array}\right) \text {, }
$$

where $\bar{c} \equiv \mathrm{e}^{\frac{1}{2} \bar{\Phi}}$. It has three wave solutions of the type $\mathbf{X}(x, t)=$ $\hat{\mathbf{X}} \exp [i(k x-\omega t)]$ : a slow advective solution with dispersion relation $\omega_{0}=k \bar{u}$ corresponding to geostrophic balance state $(u=$ 0 , and $\left.f v=\bar{c}^{2} \partial \Phi / \partial x\right)$ and two fast solutions corresponding to gravity wave solutions with $\omega_{ \pm}=k\left(\bar{u} \pm c_{k}\right)$ and $c_{k}^{2}=\bar{c}^{2}+$ $(f / k)^{2}$. The characteristic values of this model are

$p_{a}=v, \quad p=u+\bar{c} \Phi, \quad q=u-\bar{c} \Phi$,

and their associated characteristics are $\bar{u}$ and $\bar{u} \pm \bar{c}$, respectively.

This model is integrated by a two-time-level semi-implicit (Robert, 1969) semi-Lagrangian (Robert, 1981) discretization (2TL SISL). This time discretization can formally be written as

$\left[\left(\mathbf{I}-\frac{\Delta t}{2} \mathbf{L}\right) \mathbf{X}^{+}\right]_{\mathbf{A}}=\left[\left(\mathbf{I}+\frac{\Delta t}{2} \mathbf{L}\right) \mathbf{X}^{0}\right]_{\mathbf{D}} \equiv \mathbf{R}_{\mathbf{D}}$,

where $\Delta t$ denotes the time step and matrix $\mathbf{I}$ is the $3 \times 3$ identity matrix. The superscripts + and 0 denote time level $t+\Delta t$ and $t$, respectively. Time level $t-\Delta t$ will be denoted by - . The 
subscripts A and $\mathbf{D}$, respectively, denote quantities computed at the arrival point and the departure point of the semi-Lagrangian trajectories. The departure point is given by $\mathbf{D}=\mathbf{A}-\bar{u} \Delta t$.

This scheme is unconditionally stable and second-order accurate. The right-hand side (r.h.s.) explicit terms are computed in gridpoint space, yielding values $\mathbf{R}_{\mathbf{A}}=\left(\mathbf{I}+\frac{\Delta t}{2} \mathbf{L}\right) \mathbf{X}$. These are then interpolated to the departure point $\mathbf{D}$ to yield $\mathbf{R}_{\mathbf{D}}$. For the sake of the notation, the subscripts $\mathbf{D}$ will be dropped henceforth; unless indicated by a subscript $\mathbf{A}$, all $R$ components will be meant to be taken in the departure points.

Applying $\mathbf{I}+\frac{\Delta t}{2} \mathbf{L}$ to the left-hand side (1.h.s.) and r.h.s. of (6) yields a $u$ component that has the form of a Helmholtz equation:

$$
\begin{aligned}
& {\left[1+\frac{f^{2} \Delta t^{2}}{4}-\frac{\Delta t^{2} \bar{c}^{2}}{4} \frac{\partial^{2}}{\partial x^{2}}\right] u^{+}} \\
& =R^{u}+\frac{f \Delta t}{2} R^{v}-\frac{\Delta t \bar{c}^{2}}{2} \frac{\partial R^{\Phi}}{\partial x} .
\end{aligned}
$$

This equation is independent of the variables $v^{+}$and $\Phi^{+}$and is solved to find the field $u^{+}$. The fields $v^{+}$and $\Phi^{+}$can then be obtained from (6) in a straightforward manner.

This paper will consider a gridpoint model and a spectral model version of these shallow-water equations. The corresponding space discretizations and the idea of externalization are introduced below. The details of the lateral-boundary conditions in the externalized setup will be discussed separately in detail in Section 3.

\subsection{A gridpoint version with intrinsic $L B C$ 's}

In the interior of the domain, the space discretization of (6) in the gridpoint model essentially follows McDonald (2000).

The gridpoint version of the scheme in (6) was discretized on a Arakawa C grid (Arakawa and Lamb, 1977), spanning $N+1$ grid points labelled by $I=0, \ldots, N$ located at $x_{I}=I \Delta x$, and the odd intermediate grid points label by $I+\frac{1}{2}=-\frac{1}{2}, \ldots, N+\frac{1}{2}$ located at $x_{I+\frac{1}{2}}=\left(I+\frac{1}{2}\right) \Delta x$. The points $I=0$ and $I=N$ correspond to the lefthand boundary at $x=0$ and the right-hand boundary at $x=L=N \Delta x$, respectively. The variables $\Phi_{I}$ and $v_{I}$ are located on the even grid points and the variables $u_{I+\frac{1}{2}}$ are on the odd ones. The components of $\mathbf{R}_{A}$ are discretized as

$R_{\mathbf{A}, I+\frac{1}{2}}^{u}=u_{I+\frac{1}{2}}^{0}+\frac{\Delta t f}{4}\left(v_{I+1}^{0}+v_{I}^{0}\right)-\frac{\Delta t \bar{c}^{2}}{2 \Delta x}\left(\Phi_{I+1}^{0}-\Phi_{I}^{0}\right)$,

$R_{\mathbf{A}, I}^{v}=v_{I}^{0}-\frac{\Delta t f}{4}\left(u_{I+\frac{1}{2}}^{0}+u_{I-\frac{1}{2}}^{0}\right)$,

$R_{\mathbf{A}, I}^{\Phi}=\Phi_{I}^{0}-\frac{\Delta t}{2 \Delta x}\left(u_{I+\frac{1}{2}}^{0}-u_{I-\frac{1}{2}}^{0}\right)$.

The values of the r.h.s.s are computed at the grid points and are then interpolated to the departure points of the semi-Lagrangian trajectories by means of a cubic interpolation, similarly as is done in McDonald (2000).
In this C-grid discretization, the Helmholtz eq. (7) gets the form

$$
\begin{aligned}
u_{I+\frac{1}{2}}^{+} & +\left(\frac{f \Delta t}{4}\right)^{2}\left(u_{I+\frac{3}{2}}^{+}+2 u_{I+\frac{1}{2}}^{+}+u_{I-\frac{1}{2}}^{+}\right) \\
& -\left(\frac{\Delta t \bar{c}}{2 \Delta x}\right)^{2}\left(u_{I+\frac{3}{2}}^{+}-2 u_{I+\frac{1}{2}}^{+}+u_{I-\frac{1}{2}}^{+}\right) \\
=R_{I+\frac{1}{2}}^{u} & +\frac{f \Delta t}{4}\left(R_{I+1}^{v}+R_{I}^{v}\right)-\frac{\Delta t \bar{c}^{2}}{2 \Delta x}\left(R_{I+1}^{\Phi}-R_{I}^{\Phi}\right) .
\end{aligned}
$$

The $v$ and $\Phi$ component of (6),

$v_{I}^{+}+\frac{\Delta t f}{4}\left(u_{I+\frac{1}{2}}^{+}+u_{I-\frac{1}{2}}^{+}\right)=R_{I}^{v}$,

$\Phi_{I}^{+}+\frac{\Delta t}{2 \Delta x}\left(u_{I+\frac{1}{2}}^{+}-u_{I-\frac{1}{2}}^{+}\right)=R_{I}^{\Phi}$,

are added to this to compute $v_{I}^{+}$and $\Phi_{I}^{+}$.

Considering the case $\bar{c}>\bar{u}$, a possibility is to impose the incoming characteristic values $v_{0}^{\mathrm{h}}$ and $p_{0}^{\mathrm{h}}$ at the left-hand boundary at $I=0$ and the characteristic value $q_{N}^{\mathrm{h}}$ at the right-hand boundary $I=N$ (see McDonald, 2000), where the superscript h refers to the host model. This can be done by replacing $v_{0}^{+}$by $v_{0}^{\mathrm{h}}$ and specifying the other boundary fields $p_{0}^{\mathrm{h}}$ and $q_{N}^{\mathrm{h}}$. These are then used to compute

$u_{-\frac{1}{2}}=2\left(p_{0}^{\mathrm{h}}-\bar{c} \Phi_{0}\right)-u_{\frac{1}{2}}$,

$u_{N+\frac{1}{2}}=2\left(q_{N}^{\mathrm{h}}+\bar{c} \Phi_{N}\right)-u_{N-\frac{1}{2}}$.

To have stable formulations of the LBCs, they should be imposed in the implicit part of the semi-implicit scheme, that is, by reformulating eqs. (11)-(13) at the boundaries. This leads, following McDonald (2000), to the following equations

$\left(1+\frac{\bar{c} \Delta t}{\Delta x}\right) \Phi_{0}^{+}+\frac{\Delta t}{\Delta x} u_{\frac{1}{2}}^{+}-\frac{\Delta t}{\Delta x} p_{0}^{\mathrm{h}}=R_{0}^{\Phi}$,

after substituting (14) in (13), at the left-hand boundary and, analogously,

$v_{N}^{+}+\frac{\Delta t f}{2}\left(q_{N}^{\mathrm{h}}+\bar{c} \Phi_{N}^{+}\right)=R_{N}^{v}$,

$\left(1+\frac{\bar{c} \Delta t}{\Delta x}\right) \Phi_{N}^{+}-\frac{\Delta t}{\Delta x} u_{N-\frac{1}{2}}^{+}+\frac{\Delta t}{\Delta x} q_{N}^{\mathrm{h}}=R_{N}^{\Phi}$,

at the right-hand boundary. The Helmholtz equations at the boundaries become

$$
\begin{aligned}
& {\left[1+\left(\frac{f \Delta t}{4}\right)^{2}+(1+2 \mu)\left(\frac{\bar{c} \Delta t}{2 \Delta x}\right)^{2}\right] u_{\frac{1}{2}}^{+}} \\
& +\left[\left(\frac{f \Delta t}{4}\right)^{2}-\left(\frac{\bar{c} \Delta t}{2 \Delta x}\right)^{2}\right] u_{\frac{3}{2}}^{+}=R_{\frac{1}{2}}^{u}+\frac{\bar{f} \Delta t}{4}\left(R_{1}^{v}+v_{0}^{\mathrm{h}}\right) \\
& \quad-\frac{\bar{c}^{2} \Delta t}{2 \Delta x}\left[R_{1}^{\Phi}-\mu\left(R_{0}^{\Phi}+\frac{\Delta t}{\Delta x} p_{0}^{\mathrm{h}}\right)\right]
\end{aligned}
$$


Table 1. Geometrical structure of the resolved and imposed fields and their (prognostic) equations in the gridpoint model with intrinsic LBCs

\begin{tabular}{lcccccccccccccc}
\hline & $-\frac{1}{2}$ & 0 & $\frac{1}{2}$ & 1 & $\frac{3}{2}$ & 2 & $\ldots$ & $N-\frac{3}{2}$ & $N-1$ & $N-\frac{1}{2}$ & $N$ & $N+\frac{1}{2}$ & Number \\
\hline Equation & - & $(16)$ & $(19)$ & $(12)(13)$ & $(11)$ & $(12)(13)$ & $\ldots$ & $(11)$ & $(12)(13)$ & $(20)$ & $(17)(18)$ & - & $3 N+1$ \\
Resolved variable & - & $\Phi_{0}^{+}$ & $u_{\frac{1}{2}}^{+}$ & $v_{1}^{+}$ & $u_{\frac{3}{2}}^{+}$ & $\Phi_{2}^{+}$ & $\ldots$ & $u_{N-\frac{3}{2}}^{+}$ & $\Phi_{N-1}^{+}$ & $u_{N-\frac{1}{2}}^{+}$ & $\Phi_{N}^{+}$ & - & $3 N+1$ \\
& & & & & & & & & & & & \\
Imposed variable & $(14)$ & $v_{0}^{h}$ & - & - & - & - & $\ldots$ & - & - & - & - & $(15)$ & 3 \\
\hline
\end{tabular}

and

$$
\begin{aligned}
& {\left[1+\left(1+2 \mu \frac{\bar{c} \Delta t}{\Delta x}\right)\left(\frac{f \Delta t}{4}\right)^{2}+(1+2 \mu)\left(\frac{\bar{c} \Delta t}{2 \Delta x}\right)^{2}\right] u_{N-\frac{1}{2}}^{+}+} \\
& {\left[\left(\frac{f \Delta t}{4}\right)^{2}-\left(\frac{\bar{c} \Delta t}{2 \Delta x}\right)^{2}\right] u_{N-\frac{3}{2}}^{+}=R_{N-\frac{1}{2}}^{u}+\frac{f \Delta t}{4}\left(R_{N}^{v}+R_{N-1}^{v}\right)} \\
& -\mu\left[2 \bar{c}\left(\frac{f \Delta t}{4}\right)^{2}+\frac{\bar{c}^{2} \Delta t}{2 \Delta x}\right]\left(R_{N}^{\Phi}-\frac{\Delta t}{\Delta x} q_{N}^{\mathrm{h}}\right)+\frac{\bar{c}^{2} \Delta t}{2 \Delta x} R_{N-1}^{\Phi} \\
& -2\left(\frac{\Delta t f}{4}\right)^{2} q_{N}^{\mathrm{h}},
\end{aligned}
$$

with $\mu \equiv 1 /\left(1+\frac{\bar{c} \Delta t}{\Delta x}\right)$.

As can be seen from Table 1, eqs. (12) and (13) for $I=1$, $\ldots, N-1$ and (11) for $I=1, \ldots, N-2$, together with eqs. (16)-(20), form a linear system of $3 N+1$ equations and an equal number of unknowns

$\Phi_{0}^{+}, u_{\frac{1}{2}}^{+}, v_{1}^{+}, \Phi_{1}^{+}, \ldots, u_{N-\frac{1}{2}}^{+}, v_{N}^{+}, \Phi_{N}^{+}$.

The values of $u_{-\frac{1}{2}}^{+}$and $u_{N+\frac{1}{2}}^{+}$in (14) and (15) and $v_{0}^{h}$ are added to the values in (21) to provide the input for the next time step in the eqs. (8)-(10).

For the computation of the interpolated r.h.s. terms, trajectory truncation is used, as introduced by McDonald (2000); if the departure point is such that the interpolation requires fields outside the area of integration, then the trajectory is truncated to the nearest point on the boundary.

Other combinations of $u, v$ and $\Phi$ may be used as wellposed boundary condition, see Oliger and Sundström (1978) and Sundström and Elvius (1979). Besides the ones in (14) and (15), McDonald (2000) presents tests imposing $v_{0}, \phi_{0}$ and $\phi_{N}$. Imposing such alternatives at the boundaries in eqs. (11)-(13) yields equations other than (16)-(20), with different unknowns than the ones in (21) and a different geometrical structure than the one in Table 1.

Inverting the semi-implicit (SI) operator is a non-local operation, making these LBCs 'intrinsically' linked to the formulation of the dynamics in the interior domain. Each time one decides to implement and test other conditions, for example, other fields or higher-order conditions, the form and the numerics of these equations changes. In practice, this implies that any scientific development of the LBCs and of the numerical scheme of the dynamic core become tightly linked.

\subsection{Externalizing the LBCs from the gridpoint model}

This link between the LBCs and the numerics of the interior can be removed by the following approach. For the LBCs, the code calls a separate routine that uses the fields $u_{-\frac{1}{2}}^{0}, v_{0}^{0}, \Phi_{0}^{0}, \ldots, v_{N}^{0}, \Phi_{N}^{0}, u_{N+\frac{1}{2}}^{0}$ near the boundaries, at time level $t$, as input and computes the values at time level $t+\Delta t$ :

$\left.\tilde{\mathbf{X}}_{L}^{+}\right|_{\partial D} \equiv \tilde{u}_{-\frac{1}{2}}^{+}, \tilde{\Phi}_{0}^{+}, \tilde{v}_{0}^{+}$

at the left-hand boundary and

$\left.\tilde{\mathbf{X}}_{R}^{+}\right|_{\partial D} \equiv \tilde{\Phi}_{N}^{+}, \tilde{v}_{N}^{+}, \tilde{u}_{N+\frac{1}{2}}^{+}$

at the right-hand boundary, while properly distinguishing the incoming from the outgoing modes. The boundary of the domain $D$ is represented by the points $-\frac{1}{2}, 0$ and $N, N+\frac{1}{2}$ and is formally written by $\partial$.

Three examples of schemes that compute the values in (22) and (23) will be described separately in detail in Section 3. This reflects the essence of the proposed approach of the present paper: the dynamic core is not obliged to care about the details of the extrinsic LBC scheme but must solely agree to accept the values (22)and (23) irrespective of their details.

It will be shown later that model codes can be organized such that the numerical core receives the values in (22) and (23) as input. At the boundaries the equations of the form

$$
\begin{aligned}
u_{\frac{1}{2}}^{+} & +\left(\frac{f \Delta t}{4}\right)^{2}\left(u_{\frac{3}{2}}^{+}+u_{\frac{1}{2}}^{+}\right) \\
& -\left(\frac{\Delta t \bar{c}}{2 \Delta x}\right)^{2}\left(u_{\frac{3}{2}}^{+}-u_{\frac{1}{2}}^{+}\right) \\
=R_{\frac{1}{2}}^{u} & +\frac{f \Delta t}{4}\left(R_{1}^{v}+\tilde{v}_{0}^{+}\right)-\frac{\Delta t \bar{c}^{2}}{2 \Delta x}\left(R_{1}^{\Phi}-\tilde{\Phi}_{0}^{+}\right),
\end{aligned}
$$

and

$$
\begin{aligned}
u_{N-\frac{1}{2}}^{+} & +\left(\frac{f \Delta t}{4}\right)^{2}\left(u_{N-\frac{3}{2}}^{+}+u_{N-\frac{1}{2}}^{+}\right) \\
& -\left(\frac{\Delta t \bar{c}}{2 \Delta x}\right)^{2}\left(u_{N-\frac{3}{2}}^{+}-u_{N-\frac{1}{2}}^{+}\right) \\
R_{N-\frac{1}{2}}^{u} & +\frac{f \Delta t}{4}\left(R_{N-1}^{v}+\tilde{v}_{N}^{+}\right)-\frac{\Delta t \bar{c}^{2}}{2 \Delta x}\left(\tilde{\Phi}_{N}^{+}-R_{N-1}^{\Phi}\right),
\end{aligned}
$$

are solved. In the interior, $I=1, \ldots, N-1$, the eqs. (11)-(13) are used. This is illustrated in Table 2 as opposed to Table 1. Please note that contrary to the intrinsic LBCs in (16)-(20), eq. (25) for 
Table 2. Geometrical structure of the resolved and imposed fields and their (prognostic) equations in the gridpoint model with extrinsic LBCs

\begin{tabular}{|c|c|c|c|c|c|c|c|c|c|c|c|c|c|}
\hline & $-\frac{1}{2}$ & 0 & $\frac{1}{2}$ & 1 & $\frac{3}{2}$ & 2 & $\ldots$ & $N-\frac{3}{2}$ & $N-1$ & $N-\frac{1}{2}$ & $N$ & $N+\frac{1}{2}$ & Number \\
\hline Equation & - & - & (24) & (12) (13) & (11) & (12) (13) & $\ldots$ & (11) & (12) (13) & (25) & - & - & $3 N-2$ \\
\hline Resolved variable & - & - & $u_{\frac{1}{2}}^{+}$ & $\begin{array}{c}v_{1}^{+} \\
\Phi_{1}^{+}\end{array}$ & $u_{\frac{3}{2}}^{+}$ & $\begin{array}{l}v_{2}^{+} \\
\Phi_{2}^{+}\end{array}$ & $\cdots$ & $u_{N-\frac{3}{2}}^{+}$ & $\begin{array}{l}v_{N-1}^{+} \\
\Phi_{N-1}^{+}\end{array}$ & $u_{N-\frac{1}{2}}^{+}$ & - & - & $3 N-2$ \\
\hline Extrinsic variable & $\tilde{u}_{-\frac{1}{2}}^{+}$ & $\begin{array}{l}\tilde{v}_{0}^{+} \\
\tilde{\Phi}_{0}^{+}\end{array}$ & - & - & - & - & $\ldots$ & - & - & - & $\begin{array}{c}\tilde{v}_{N}^{+} \\
\tilde{\Phi}_{N}^{+}\end{array}$ & $\tilde{u}_{N+\frac{1}{2}}^{+}$ & 6 \\
\hline
\end{tabular}

the externalized LBCs at the right-hand boundary is symmetric to eq. (24) at the left-hand boundary.

It will be shown in Section 4.3 that improved accuracy can be obtained if the externalized LBC scheme computes more values than in (22), as given by

$\tilde{u}_{-\frac{1}{2}}^{+}, \tilde{\Phi}_{0}^{+}, \tilde{v}_{0}^{+}, \tilde{u}_{\frac{1}{2}}^{+}, \tilde{\Phi}_{1}^{+}, \tilde{v}_{1}^{+}, \ldots, \tilde{u}_{N_{\text {buf }}-\frac{1}{2}}^{+}, \tilde{\Phi}_{N_{\text {buf }}}^{+}, \tilde{v}_{N_{\text {buf }}}^{+}$

in a buffer zone of $N_{\text {buf }}$ points near the left-hand boundary. This vector will be denoted as $\tilde{\mathbf{X}}_{L}^{+}$henceforth. In that case, after the computation of $\mathbf{X}^{+}$by solving the implicit part, the values near the boundary may be replaced by these values in (26). This leads to a wider buffer zone of values computed by the LBC scheme. The same holds for

$$
\begin{aligned}
\tilde{\mathbf{X}}_{R}^{+}= & \tilde{\Phi}_{N}^{+}, \tilde{v}_{N}^{+}, \tilde{u}_{N+\frac{1}{2}}^{+} \tilde{\Phi}_{N-1}^{+}, \tilde{v}_{N-1}^{+}, \tilde{u}_{N-\frac{1}{2}}^{+}, \ldots, \\
& \tilde{\Phi}_{N-N_{\text {buf }}}^{+}, \tilde{v}_{N-N_{\text {buf }}}^{+}, \tilde{u}_{N-N_{\text {buf }}+\frac{1}{2}}^{+}
\end{aligned}
$$

in a buffer zone near the right-hand boundary. The definitions (22) and (23) are particular cases of (26)and (27) for $N_{\text {buf }}=0$.

The details of the data flow of the externalized LBCs in a gridpoint model is shown in Fig. 1. The dynamic core is displayed at the left-hand part of the scheme and the extrinsic LBC scheme is at the right-hand part. The communication between the two schemes is indicated by the double-lined arrows. Both schemes use the model states at times $t$ and $t-\Delta t$. The explicit part of the dynamic core and the extrinsic LBC scheme are computed in an entirely separate way. The parts $\left.\tilde{\mathbf{X}}_{L}^{+}\right|_{\partial D}$ and $\left.\tilde{\mathbf{X}}_{R}^{+}\right|_{\partial D}$ of $\tilde{\mathbf{X}}_{L}^{+}$and $\tilde{\mathbf{X}}_{R}^{+}$are then used to solve eqs. (24) and (25) as conventionally symbolized by the leftward double-lined arrow. Additionally, the use of the buffer zone is indicated by the dashed double-lined arrow. For this option, the values of $\mathbf{X}^{+}$near the boundaries are simply replaced by $\tilde{\mathbf{X}}_{L}^{+}$and $\tilde{\mathbf{X}}_{R}^{+}$at the end of the time-step computation while all the other computations remain the same.

In Section 3, it will be shown how the values near the boundaries in (26) and (27) can be computed to yield an LBC formulation with an accuracy and stability that is comparable to the intrinsic LBCs.

\subsection{A spectral model}

This paper also considers a spectral-model version of the shallow-water eqs. (1)-(3) with a structure, following the proposal of Haugen and Machenhauer (1993). This model is discretized on a collocation A grid with index $I=0, \ldots, N$, index

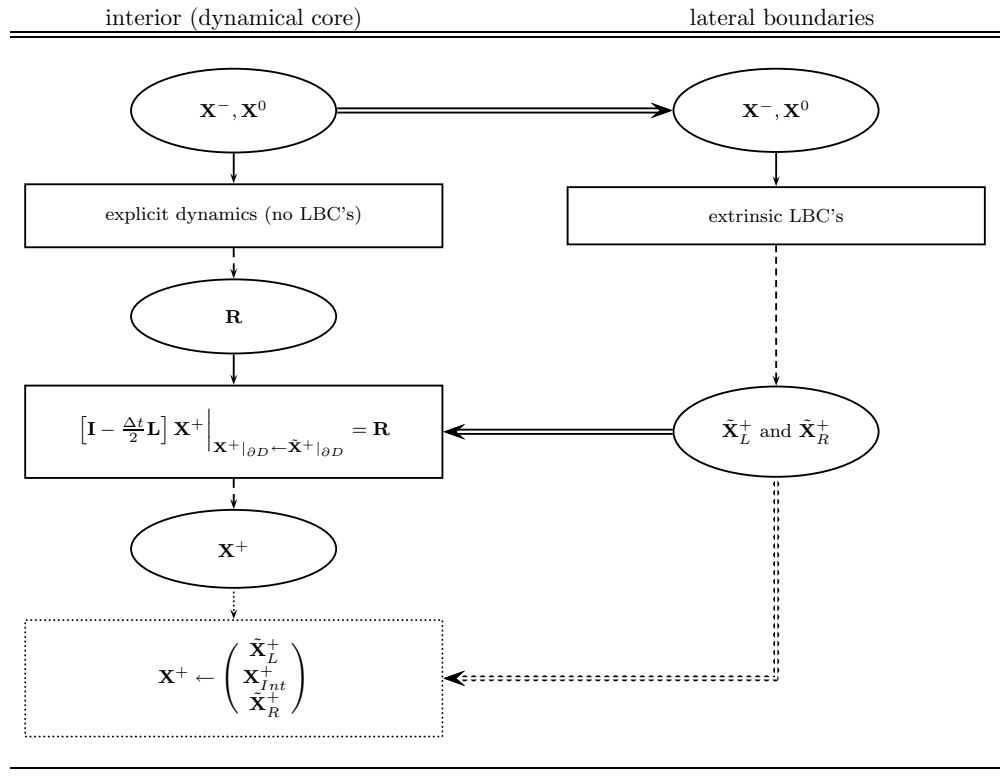

Fig. 1. Data flow in the gridpoint version for the externalized LBCs. Rectangular boxes represent numerical operations, solid single-lined arrows represent input and dashed single-lined arrows represent output. Double-lined arrows represent the communication between the dynamic core and the extrinsic LBC scheme. The dashed double-lined arrow represents the option to impose an extrinsic buffer zone with $N_{\text {buf }}>0$. 
$I=0$ representing the left-hand side artificial boundary and $I=$ $N$, the right-hand boundary. This physical area is extended by an artificial extension zone (called the E-zone) labelled by $I=$ $N+1, \ldots, L-1$ making the domain periodic (i.e. the point $x=$ $L \Delta x$ is identified with the point $x=0$ ). In this zone, all fields are supplemented with a periodic extension by means of a spline interpolation, before applying the Fourier transforms. This spline is exactly the same as the existing one in the ALADIN model.

At the beginning of the time step computation, the fields $\mathbf{X}^{0}$ are present in spectral space and the derivatives are computed thereof. Then an inverse Fourier transform is taken of the fields and their derivatives to proceed in gridpoint space. The expression for $\mathbf{R}_{\boldsymbol{A}}$ on the A grid is then computed as

$R_{\mathbf{A}, I}^{u}=u_{I}^{0}+\frac{\Delta t}{2} f v_{I}^{0}-\frac{\Delta t \bar{c}^{2}}{2} \hat{\partial} \Phi_{I}^{0}$,

$R_{\mathrm{A}, I}^{v}=v_{I}^{0}-\frac{\Delta t}{2} f u_{I}^{0}$,

$R_{\mathbf{A}, I}^{\Phi}=\Phi_{I}^{0}-\frac{\Delta t}{2} \hat{\partial} u_{I}^{0}$,

where the derivative $\hat{\partial}$ is $\partial / \partial x$ that has been computed in spectral space. Then these values are also interpolated to the departure points to yield $R_{I}^{u}, R_{I}^{v}$ and $R_{I}^{\Phi}$.

The Helmholtz eq. (7) is solved by inverting the differential operator on the 1.h.s. of (7), which in spectral space is obtained by a simple algebraic division, that is,

$\hat{u}_{K}^{+}=\frac{\hat{R}_{K}^{u}+\frac{\Delta t}{2} f \hat{R}_{K}^{v}-\frac{\Delta t \bar{c}^{2}}{2} \frac{2 \pi i K}{\mathcal{L}} \hat{R}_{K}^{\Phi}}{1+\frac{\Delta t^{2}}{4} f^{2}-\frac{\Delta t^{2} \bar{c}^{2}}{4}\left(\frac{2 \pi i K}{\mathcal{L}}\right)^{2}}$,

where $\hat{u}_{K}^{+}, \hat{R}_{K}^{u}, \hat{R}_{K}^{v}$ and $\hat{R}_{K}^{\Phi}$ are the spectral coefficients corresponding with wavenumber $K$ of $u^{+}, R^{u}, R_{v}$ and $R^{\Phi}$, respectively, and $2 \pi i K / \mathcal{L}$ the multiplicative factor of the derivative $\hat{\partial}$, with $\mathcal{L}=L \Delta x$. The equations for $\hat{v}_{K}^{+}$and $\hat{\Phi}_{K}^{+}$are then easily solved by substitution.

Due to the non-local action of the exact derivatives computed as in (31), the period extension by the spline may still create a certain degree of contamination of the extension zone into the physical interior zone when solving the Helmholtz equation. The experience in the operational ALADIN models has shown that this stays within acceptable levels. More exact methods, such as the one proposed by Boyd (2005), will not be investigated here. In this paper, the analysis will be restricted to the method used in the ALADIN model.

Imposing the lateral boundary conditions locally at a specific point is not possible in the implicit part of the scheme, that is, by modifying the Helmholtz eq. (31) in spectral space as it has been done in the gridpoint model in equations (19) and (20). Imposing them in the explicit part of the scheme, in (28)-(30), leads to a scheme that becomes unstable for too short time steps, to be of operational interest.

It is possible to use the output of the same scheme as the one used for the gridpoint model on the $\mathrm{C}$ grid, as provided in (26) and (27). To this end, the following objects are computed:

$\tilde{R}_{I}^{u}=\frac{1}{2}\left(\tilde{u}_{I-\frac{1}{2}}^{+}+\tilde{u}_{I+\frac{1}{2}}^{+}\right)-\frac{\Delta t f}{2} \tilde{v}_{I}^{+}+\frac{\Delta t \bar{c}^{2}}{4 \Delta x}\left(\tilde{\Phi}_{I+1}^{+}-\tilde{\Phi}_{I-1}^{+}\right)$,

$\tilde{R}_{I}^{v}=\tilde{v}_{I}^{+}+\frac{\Delta t f}{4}\left(\tilde{u}_{I+\frac{1}{2}}^{+}+\tilde{u}_{I-\frac{1}{2}}^{+}\right)$,

$\tilde{R}_{I}^{\Phi}=\tilde{\Phi}_{I}^{0}+\frac{\Delta t}{2 \Delta x}\left(\tilde{u}_{I+\frac{1}{2}}^{+}-\tilde{u}_{I-\frac{1}{2}}^{+}\right)$

and

$\tilde{R}_{0}^{u}=\frac{1}{2}\left(\tilde{u}_{-\frac{1}{2}}^{+}+\tilde{u}_{\frac{1}{2}}^{+}\right)-\frac{\Delta t f}{2} \tilde{v}_{0}^{+}+\frac{\Delta t \bar{c}^{2}}{2 \Delta x}\left(\tilde{\Phi}_{1}^{+}-\tilde{\Phi}_{0}^{+}\right)$,

$\tilde{R}_{0}^{v}=\tilde{v}_{0}^{+}+\frac{\Delta t f}{4}\left(\tilde{u}_{\frac{1}{2}}^{+}+\tilde{u}_{-\frac{1}{2}}^{+}\right)$

$\tilde{R}_{0}^{\Phi}=\tilde{\Phi}_{0}^{0}+\frac{\Delta t}{2 \Delta x}\left(\tilde{u}_{\frac{1}{2}}^{+}-\tilde{u}_{-\frac{1}{2}}^{+}\right)$

at the left-hand boundary. Since $\Phi_{-1}$ lies beyond the boundary, the decentred derivative is taken in (35). The equations are mirrored at the right-hand boundary. For a buffer zone of width $N_{\text {buf }} \Delta x$, this allows to compute $\tilde{R}_{0}, \tilde{R}_{1}, \ldots, \tilde{R}_{N_{\text {buf }}-1}$. This can formally be written as,

$\tilde{\mathbf{R}} \equiv\left(\mathbf{I}-\frac{\Delta t}{2} \mathbf{L}\right)_{F D} \tilde{\mathbf{X}}^{+}$

where $\tilde{\mathbf{X}}^{+}$are the values interpolated to the A grid, obtained by an externalized treatment of the LBCs in either (26) or (27). The subscript $F D$ denotes that the derivatives in this operators are computed as finite differences in gridpoint space. Subsequently the values of $\mathbf{R}$ are replaced by $\tilde{\mathbf{R}}$ at $I=0$ and $I=N$. Solving the Helmholtz equation and the equations for $u$ and $\Phi$ is formally equivalent to the $\left[\mathbf{I}-\frac{\Delta t}{2} \mathbf{L}\right]_{S P}^{-1}$ operator with $S P$ denoting that the inversion is performed in spectral space. This will approximatively yield the values of $\left.\tilde{\mathbf{X}}_{L}^{+}\right|_{\partial D}$ and $\left.\tilde{\mathbf{X}}_{R}^{+}\right|_{\partial D}$, at the end of the time step.

Figure 2 shows how the computation of the extrinsic LBCs can be organized in a spectral model. As in the subgridpoint model in Fig. 1, the extrinsic scheme is computed parallelly to the explicit part of the dynamic core. The difference is that the LBC scheme computes $\tilde{\mathbf{R}}$ as in (38). The leftward arrow corresponds to replacing the obtained $\mathbf{R}$ by $\mathbf{R}_{L}$ and $\mathbf{R}_{R}$ near the boundaries before going to spectral space. In the spectral model, a wider buffer zone can also be included by using $N_{\text {buf }}>1$ and replacing $\mathbf{R}$ by $\tilde{\mathbf{R}}$ in more than one grid point near the boundary.

The properties of the scheme will depend on the numerical properties of the operator

$\mathbf{Q}=\left(1-\frac{\Delta t}{2} \mathbf{L}\right)_{S P}^{-1}\left(1-\frac{\Delta t}{2} \mathbf{L}\right)_{F D}$ 


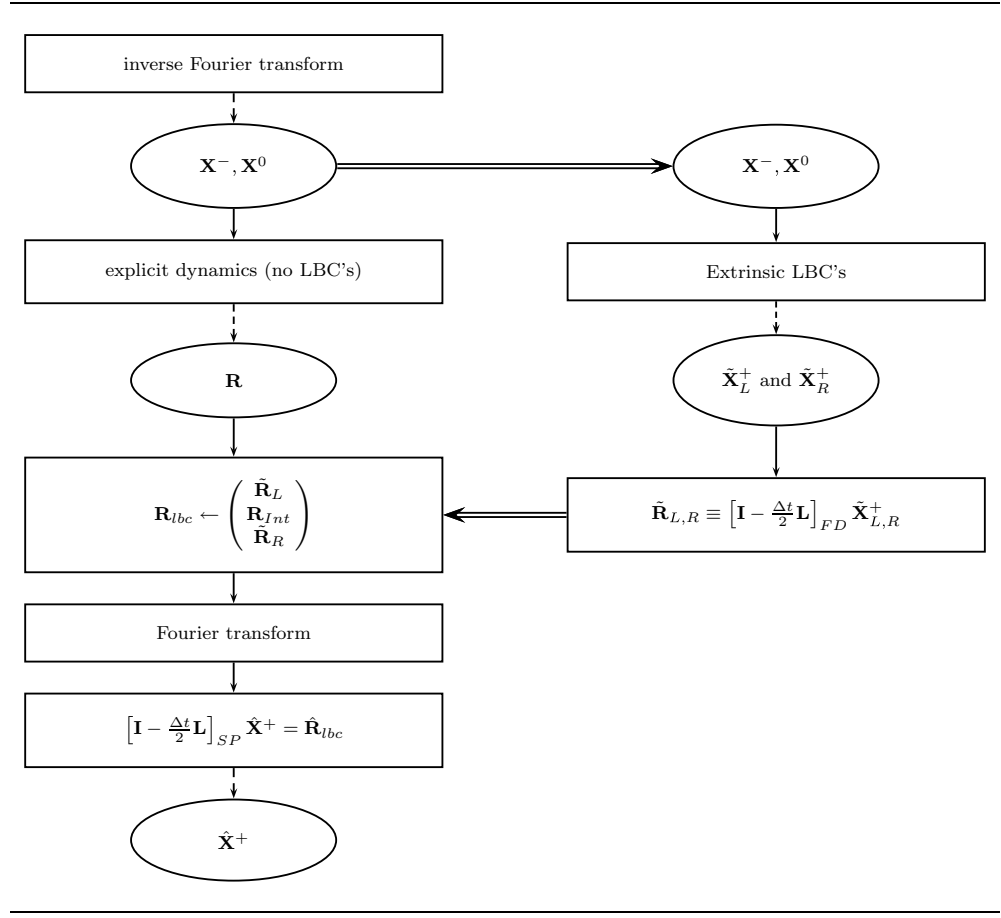

Fig. 2. Data flow in the spectral version for the externalized LBCs. The same conventions are used as in Fig. 1.

They can be studied by considering the mode $\psi_{I}(\xi)=\mathrm{e}^{i I \xi}$, with $\xi=2 \pi \frac{K \Delta x}{\mathcal{L}}$,

and where $I$ denotes the gridpoint location. The components of $\mathbf{Q}$ are

$Q_{11}=\frac{4 \cos \left(\frac{\xi}{2}\right) \bar{c}^{2}+\sigma^{2}\left[2 \xi \sin \left(\frac{\xi}{2}\right) \bar{c}^{2}+\Delta x^{2} f^{2}\right]}{\left(\sigma^{2} \xi^{2}+4\right) \bar{c}^{2}+\Delta x^{2} f^{2} \sigma^{2}}$,

$Q_{12}=0$,

$Q_{13}=\frac{2 \bar{c}^{3} \sigma\left(-i \xi+\mathrm{e}^{i \xi}-1\right)}{\left(\sigma^{2} \xi^{2}+4\right) \bar{c}^{2}+\Delta x^{2} f^{2} \sigma^{2}}$,

$Q_{21}=\frac{\bar{c} \Delta x f \sigma\left[\xi^{2} \sigma^{2}-2 \xi \sin \left(\frac{\xi}{2}\right) \sigma^{2}-4 \cos \left(\frac{\xi}{2}\right)+4\right]}{2\left[\left(\sigma^{2} \xi^{2}+4\right) \bar{c}^{2}+\Delta x^{2} f^{2} \sigma^{2}\right]}$,

$Q_{22}=1$,

$Q_{23}=\frac{\Delta x f \sigma^{2} \bar{c}^{2}\left(i \xi-\mathrm{e}^{i \xi}+1\right)}{\Delta x^{2} f^{2} \sigma^{2}+\xi^{2} \sigma^{2} \bar{c}^{2}+4 \bar{c}^{2}}$,

$Q_{31}=$

$-\frac{i \sigma\left[4 \xi \cos \left(\frac{\xi}{2}\right) \bar{c}^{2}+\Delta x^{2} f^{2} \sigma^{2} \xi-2\left(4 \bar{c}^{2}+\Delta x^{2} f^{2} \sigma^{2}\right) \sin \left(\frac{\xi}{2}\right)\right]}{2 \bar{c}\left[\left(\sigma^{2} \xi^{2}+4\right) \bar{c}^{2}+\Delta x^{2} f^{2} \sigma^{2}\right]}$,

$Q_{32}=0$,

$Q_{33}=\frac{\left[4-i\left(-1+\mathrm{e}^{i \xi}\right) \sigma^{2} \xi\right] \bar{c}^{2}+\Delta x^{2} f^{2} \sigma^{2}}{\left(\sigma^{2} \xi^{2}+4\right) \bar{c}^{2}+\Delta x^{2} f^{2} \sigma^{2}}$,

with $\sigma=\bar{c} \Delta t / \Delta x$. The eigenvalues of $\mathbf{Q}$ are all smaller than 1. Figure 3 shows them in the interval $\left[-\frac{\pi}{3}, \frac{\pi}{3}\right]$ for the values $\sigma=10, \Delta x=10 \mathrm{~km}, f=10^{-4} \mathrm{~s}^{-1}$ and $\bar{c}=300 \mathrm{~m} \mathrm{~s}^{-1}$, which

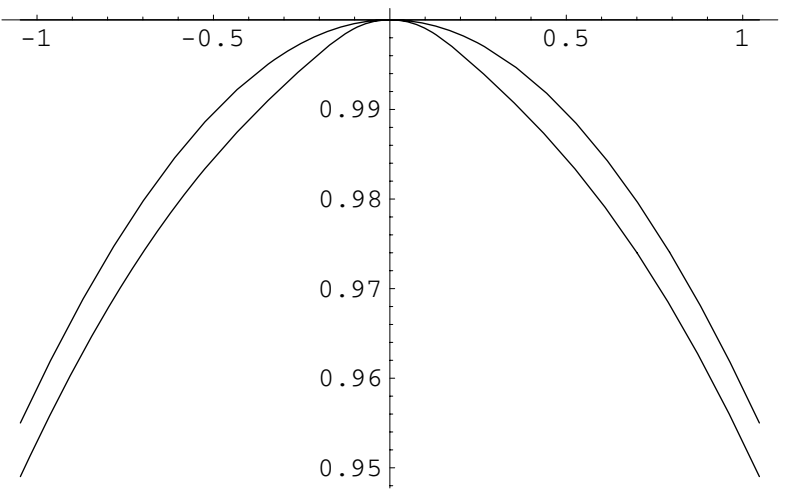

Fig. 3. The eigenvalues of $\mathbf{Q}$ for $\sigma=10, \Delta x=10 \mathrm{~km}, f=10^{-4} \mathrm{~s}^{-1}$ and $\bar{c}=300 \mathrm{~m} \mathrm{~s}^{-1}$ as a function of $\xi$ in the interval $[-\pi / 3, \pi / 3]$.

will be used later in this paper. This guarantees stability of the scheme. Ideally, the $\mathbf{Q}$ operator should be equal to $\mathbf{I}$, but due to the necessity to compute it partly in gridpoint space and partly in spectral space, it can never be exactly so. The accuracy of the approximation of unity can be studied by an expansion in terms of $\xi$. For the chosen finite differences discretization in (35)-(37), this becomes,

$$
\begin{aligned}
\mathbf{Q}= & \mathbf{I}+\frac{1}{4 \bar{c}^{2}+\Delta x^{2} f^{2} \sigma^{2}}\left(\begin{array}{ccc}
-\frac{1}{2} \bar{c}^{2} \xi^{2} & 0 & -\bar{c}^{3} \sigma \xi^{2} \\
\frac{1}{4} \bar{c} \Delta x f \sigma \xi^{2} & 0 & \frac{1}{2} \Delta x f \sigma^{2} \bar{c}^{2} \xi^{2} \\
0 & 0 & 0
\end{array}\right) \\
& +O\left(\xi^{3}\right),
\end{aligned}
$$


which is only first-order accurate in $\xi$, being mostly determined by the decentred derivative in (35).

There is an important subtlety to be addressed. The operator $\mathbf{I}-\frac{\Delta t}{2} \mathbf{L}$ has a null space of non-zero fields $\left(\mathbf{I}-\frac{\Delta t}{2} \mathbf{L}\right) \mathbf{X}^{\text {null }}=0$. For the continuous version of the operator taken, as in (4), these are the two modes given by

$$
\begin{aligned}
& u_{\lambda_{ \pm}}^{\text {null }}(x)=U \mathrm{e}^{\lambda_{ \pm} x} \\
& v_{\lambda_{ \pm}}^{\text {null }}(x)=-\frac{f \Delta t}{2} U \mathrm{e}^{\lambda_{ \pm} x} \\
& \Phi_{\lambda_{ \pm}}^{\text {null }}(x)=-\lambda_{ \pm} \frac{\Delta t}{2} U \mathrm{e}^{\lambda_{ \pm} x}
\end{aligned}
$$

with

$$
\lambda_{ \pm}= \pm \sqrt{\frac{1+\left(\frac{f \Delta t}{2}\right)^{2}}{\left(\frac{\bar{c} \Delta t}{2}\right)^{2}}} .
$$

If $\tilde{\mathbf{X}}$ contains a linear combination of these two modes, say $\tilde{\mathbf{X}}=\tilde{\mathbf{Y}}+\alpha_{+} \mathbf{X}_{\lambda_{+}}^{\text {null }}+\alpha_{-} \mathbf{X}_{\lambda_{-}}^{\text {null }}$, then they will be filtered when going to spectral space by the operator in (39), $\mathbf{Q}\left(\tilde{\mathbf{Y}}+\alpha_{+} \mathbf{X}_{\lambda_{+}}^{\text {null }}+\right.$ $\left.\alpha_{-} \mathbf{X}_{\lambda_{-}}^{\text {null }}\right)=\mathbf{Q} \tilde{\mathbf{Y}}$, so that $\tilde{\mathbf{Y}}$ will be effectively imposed rather than $\tilde{\mathbf{X}}$.

Whereas a periodic spectral model with no LBCs can not have such a mode since it is not periodic, the periodic extension with imposed LBCs could allow for it in the physical interior domain. In the present schemes where three degrees of freedom corresponding to the outgoing characteristics are not constrained by the LBCs, the possibility a priori exists that such a null mode starts to spuriously grow at the boundaries. However, the tests in Section 4 suggest that, in typical meteorological situations, it will remain acceptably small.

So, the spectral version of the externalized LBCs may be less accurate for two reasons: (1) the $\mathbf{Q}$ operator is an approximation of unity, leading to a reduced accuracy as illustrated by eq. (42), and (2) it has a null-space mode, which potentially might start to grow and propagate.

The idea of externalizing the LBC treatment, thus, becomes attractive for two reasons: (1) in gridpoint models, it may provide a way to decouple the LBCs from the development of the dynamic core, and (2) in spectral models, it may additionally provide a way around the impossibility of imposing local LBCs within the Helmholtz equation.

In the next section, it will be discussed how the fields in (26) and (27) can be computed. To test the independence of the results of the details of the chosen LBC schemes, three schemes will be considered.

\section{Some extrinsic lateral-boundary schemes}

The three 'extrinsic' schemes are chosen to be fundamentally different from the 2TL SISL in the interior domain: (1) they are explicit; (2) two Eulerian schemes will be tested alongside a semi-Lagrangian scheme; (3) a three-time-level scheme will be tested besides a two-time-level one and (4) also two predictor/corrector schemes will be considered.

The choice for explicit extrinsic schemes is motivated, additionally, by the difficulty of integrating an implicit scheme near the boundaries. Indeed, the inverse operator $\left[\mathbf{I}-\frac{\Delta t}{2} \mathbf{L}\right]^{-1}$ for the semi-implicit scheme acts non-locally over the whole domain. The idea of externalizing the LBCs becomes attractive if they can be treated near or, at most, in a small zone near the boundary.

To avoid this problem, this paper will be restricted to testing explicit schemes. The problem of the stability being limited by the CFL criterion will be overcome by resorting to substepping. On the other hand, as will be shown below, this provides an extra advantage_-substepping allows to solve the problem of truncation of the semi-Lagrangian trajectories near the boundaries.

Imposing the characteristic values in the same manner as in (14) and (15), gives the best results, see McDonald (2000). It is the purpose here to test the effect of the externalization and not to assess the particular behaviour of the choice of the imposed values. Therefore (14) and (15) will also be the choice for the extrinsic LBCs.

In the following, all schemes will be illustrated at the righthand boundary. Apart from the difference in the imposed characteristic values, the treatment of the left-hand boundary is entirely symmetric to this.

\subsection{The leapfrog scheme}

The CFL stability condition for the Eulerian leapfrog (LF) scheme is given by $(\bar{u}+\bar{c})(\Delta t / \Delta x)<1 / 2$. Thus, we ensure stability if we perform

$N_{\tau}=1+[2(\bar{u}+\bar{c})(\Delta t / \Delta x)]$

substeps of $\Delta t$, with time step $\tau=\Delta t / N_{\tau}$. In this equation, the square brackets [.] denote the integer part. The eqs. (1)-(3) are discretized on the $\mathrm{C}$ grid as

$u_{I+\frac{1}{2}}^{+\tau}=u_{I+\frac{1}{2}}^{-\tau}+2 \tau \mathcal{T}_{I+\frac{1}{2}}^{u}\left[\mathbf{X}^{0 \tau}\right]$

$v_{I}^{+\tau}=v_{I}^{-\tau}+2 \tau \mathcal{T}_{I}^{v}\left[\mathbf{X}^{0 \tau}\right]$

$\Phi_{I}^{+\tau}=\Phi_{I}^{-\tau}+2 \tau \mathcal{T}_{I}^{\Phi}\left[\mathbf{X}^{0 \tau}\right]$,

with the tendencies given by

$\mathcal{T}_{I+\frac{1}{2}}^{u}[\mathbf{X}]=-\bar{u} \frac{u_{I+\frac{3}{2}}-u_{I-\frac{1}{2}}}{2 \Delta x}+f \frac{v_{I+1}+v_{I}}{2}-\mathrm{e}^{\bar{\Phi}} \frac{\Phi_{I+1}-\Phi_{I}}{\Delta x}$,

$\mathcal{T}_{I}^{v}[\mathbf{X}]=-\bar{u} \frac{v_{I+1}-v_{I-1}}{2 \Delta x}-f \frac{u_{I+\frac{1}{2}}+u_{I-\frac{1}{2}}}{2}$,

$\mathcal{T}_{I}^{\Phi}[\mathbf{X}]=-\bar{u} \frac{\Phi_{I+1}-\Phi_{I-1}}{2 \Delta x}-\frac{u_{I+\frac{1}{2}}-u_{I-\frac{1}{2}}}{\Delta x}$,

where $-\tau, 0 \tau$ and $+\tau$ correspond, respectively, to the time step $t+(n-1) \tau, t+n \tau$ and $t+(n+1) \tau$, after $n$ substeps. 


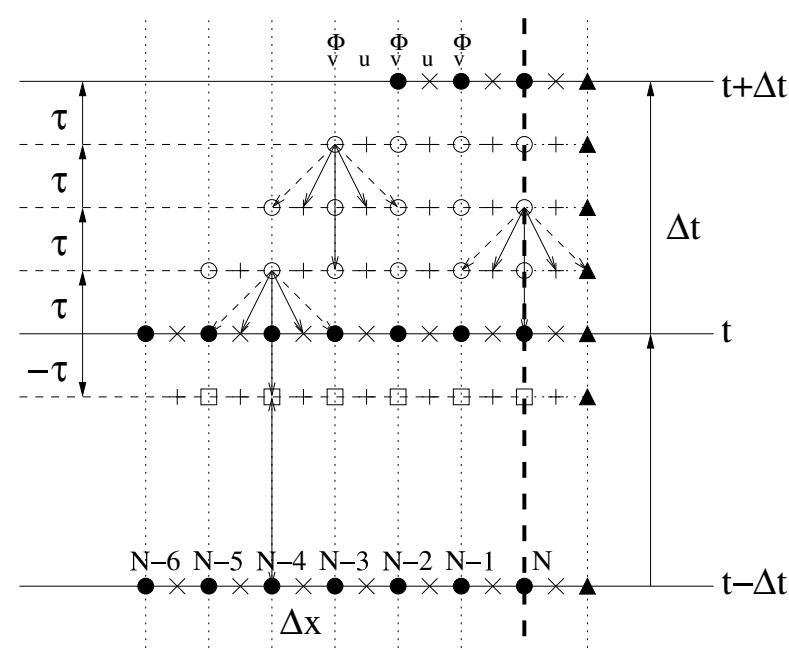

Fig. 4. The space-time structure of the extrinsic leapfrog scheme near the right-hand boundary. Space and time are represented by the horizontal and the vertical axis, respectively. The lateral boundary is represented by the thick vertical dashed line through point $N$. The fields $v$ and $\Phi$ are lying on the even grid points indicated by circles; filled ones at the $\Delta t$ time levels and empty ones at the $\tau$ substeps. The odd grid points accommodating the $u$ field, are indicated by crosses on the $\Delta t$ time levels and pluses on the substep time levels. The fields are extrapolated outside the boundary (to the triangles). The space-time dependences of the leapfrog scheme for the $\Phi$ and $v$ fields is indicated for three points $[(N-4, t+\tau),(N-3, t+3 \tau)$ and $(N, t+2 \tau)]$ by the arrows. The empty rectangles represent values that have been obtained by interpolation between the values at time level $t-\Delta t$ and $t$, needed to have the $(N-4, t-\tau)$ value to compute the first substep.

Moreover, to compute the values at $(I, t+(n+1) \tau)$ (Space time points are shorthandly written by its gridpoint index and time by time), the leapfrog scheme with second-order centred derivatives for the advection terms uses the values at $(I+1, t+$ $n \tau),(I, t+n \tau),(I-1, t+n \tau)$ and $(I, t+(n-1) \tau)$. Similarly, the values at $(I-1 / 2, t+n \tau),(I+1 / 2, t+n \tau),(I+3 / 2, t+$ $n \tau)$ and $(I+1 / 2, t+n(\tau-1))$ are needed for the computation at $(I+1 / 2, t+(n+1) \tau)$, for $n=1, \ldots, N_{\tau}$. Figure 4 illustrates this for the computation of the fields $\Phi$ and $v$. As can be seen from that figure, this leads to a triangular structure of the needed points between $t$ and $t+\Delta t$. To produce the values in (27), it is necessary to start on a buffer zone of width $N_{\text {buf }}+N_{\tau}$ at time $t$. At time $t$, the buffer zone at the right-hand side of the domain spans the points $N_{\text {int }}=N-\left(N_{\tau}+N_{\text {buf }}\right)$ to $N$. For the substeps $t<t+n \tau \leq t+\Delta t$, the buffer shrinks, covering the subdomain $N-\left(N_{\tau}+N_{\text {buf }}\right)+n, \ldots, N$.

The values from eqs. (46)-(48) at time $t+\Delta t$ are then identified as $\tilde{\mathbf{X}}_{L}^{+}$and $\tilde{\mathbf{X}}_{R}^{+}$.

It will be assumed here that only the values of $\mathbf{X}^{-}$from the previous time step and $\mathbf{X}^{0}$ are available at the full time levels (Storing the values at, for instance, time $t-\Delta t+(n-1) \tau$ in the dynamic core is in contradiction with the idea of externalizing the LBC treatment.). For the first substep, the values at $t-\tau$ are needed on the $N_{\tau}+N_{b}$ grid points near the boundaries. Here, they are provided by a linear interpolation between points at $\mathbf{X}^{-}$ and $\mathbf{X}^{0}$. This is indicated in Fig. 4 by the empty rectangles.

A Robert (1966) filter is used to control the computational mode, with a filter coefficient $\epsilon=0.067$.

Assuming that $0<\bar{u}<\bar{c}$, the characteristic boundary fields $p(0, t+(n+1) \tau), v(0, t+(n+1) \tau)$ and $q(N, t+(n+1) \tau)$ are imposed by simply overwriting the values of $u_{-\frac{1}{2}}, v_{0}$ and $u_{N+\frac{1}{2}}$ as in (14)-(15).

Outside the domain, the extrapolated values $\mathbf{X}_{-1}=2 \mathbf{X}_{0}-\mathbf{X}_{1}$ and $\mathbf{X}_{N+1}=2 \mathbf{X}_{N}-\mathbf{X}_{N-1}$ are used. The external large-scale solutions are required at each substep $t+n \tau$. From the dynamic core, they are only available at times $t+\Delta t, t$ and possibly at time $t-\Delta t$. Thus, we estimate $\mathbf{X}^{h}(x, t+n \tau)$ using quadratic interpolations between $\mathbf{X}^{h}(x, t+\Delta t), \mathbf{X}^{h}(x, t)$ and $\mathbf{X}^{h}(x, t-$ $\Delta t)$.

\subsection{The leapfrog-trapezoidal scheme}

In the case of the leapfrog-trapezoidal (LFTR) scheme, eqs. (46)(48) are used to compute a predictor $\mathbf{X} *$ which is then corrected by a corrector step given by

$u_{I+\frac{1}{2}}^{+\tau}=u_{I+\frac{1}{2}}^{0 \tau}+\frac{\tau}{2}\left(\mathcal{T}_{I}^{u}\left[\mathbf{X}^{0 \tau}\right]+\mathcal{T}_{I}^{u}\left[\mathbf{X}^{*}\right]\right)$

$v_{I}^{+\tau}=v_{I}^{0 \tau}+\frac{\tau}{2}\left(\mathcal{T}_{I}^{v}\left[\mathbf{X}^{0 \tau}\right]+\mathcal{T}_{I}^{v}\left[\mathbf{X}^{*}\right]\right)$,

$\Phi_{I}^{+\tau}=\Phi_{I}^{0 \tau}+\frac{\tau}{2}\left(\mathcal{T}_{I}^{\Phi}\left[\mathbf{X}^{0 \tau}\right]+\mathcal{T}_{I}^{\Phi}\left[\mathbf{X}^{*}\right]\right)$

This scheme dampens the computational mode; so, no Robert filter is needed (Durran, 1999). The characteristic values are imposed after the predictor step of the leapfrog scheme and after the corrector scheme, again as in (14) and (15). This scheme is stable if

$N_{\tau}=1+[\sqrt{2}(\bar{u}+\bar{c})(\Delta t / \Delta x)]$

substeps are taken.

This corrector step needs the same space dependence as the predictor step; so, the buffer zone needs to be $N_{\text {buf }}+2 N_{\tau}$.

\subsection{An iterative semi-Lagrangian explicit scheme}

Thirdly, a substepping scheme will be used, which is more closely related to the full 2TL SISL scheme used by the dynamic core of the model. It is an iterative semi-Lagrangian (ISL) explicit scheme, which is essentially a decentred version of the schemes in Bénard (2003). Keeping it explicit enables to circumvent the inversion of the linear semi-implicit correction during 
each time integration. The iterations are computed as,

$$
\begin{aligned}
u_{I+\frac{1}{2}}^{(k+1)}= & R_{I+\frac{1}{2}}^{u} \\
& +\frac{\tau}{2}\left(f \frac{v_{I+1}^{(k)}+v_{I}^{(k)}}{2}-\mathrm{e}^{\bar{\Phi}} \frac{\Phi_{I+1}^{(k)}-\Phi_{I}^{(k)}}{\Delta x}\right), \\
v_{I}^{(k+1)}= & R_{I}^{v}-\frac{\tau}{2} f\left(\frac{u_{I+\frac{1}{2}}^{(k)}+u_{I-\frac{1}{2}}^{(k)}}{2}\right), \\
\Phi_{I}^{(k+1)}= & R_{I}^{\Phi}-\frac{\tau}{2}\left(\frac{u_{I+\frac{1}{2}}^{(k)}-u_{I-\frac{1}{2}}^{(k)}}{\Delta x}\right),
\end{aligned}
$$

for $k=0,1, \mathbf{X}^{(0)}=\mathbf{X}^{0 \tau}, \tilde{\mathbf{X}}^{(+\tau)}=\mathbf{X}^{(2)}$ and where the $R$ components of the explicit part are computed identically as in (8)-(10) but with $\Delta t$ replaced by $\tau$ and interpolated to the departure point $\mathbf{D}=\mathbf{A}-\bar{u} \tau$. In principle, $\mathbf{D}$ should be recomputed each iteration, but since $\bar{u}$ is constant, this is not needed for the scheme here. The trajectories are truncated as in McDonald (2000), as already mentioned in Section 2.1. In the case of substepping, they are not only truncated at the lateral boundary but also have to be truncated in the inside boundary of the shrinking buffer on which the extrinsic substepping is performed. Besides, SL interpolations are peformed in such a way that no points outside the substepping buffer zone are involved. This implies the use of quadratic Lagrangian interpolations when the departure point is immediately adjacent to the inside boundary of the substepping buffer zone.

It can be shown that this scheme is stable provided that $\tau \bar{c} / \Delta x<1 / 2$. Therefore, by taking

$N_{\tau}=1+[2 \bar{c} \Delta t / \Delta x]$,

and $\tau=\Delta t / N_{\tau}$, it is ensured that this CFL condition is fulfilled. The wave CFL constraint is imposed by the fastest gravity modes with phase speeds of typical values of $300 \mathrm{~m} \mathrm{~s}^{-1}$, whereas the advective CFL is defined by the jet-like wind pattern with velocity around $100 \mathrm{~m} \mathrm{~s}^{-1}$. Therefore, the advection Courant number always satisfies $\alpha_{\tau}=\bar{u} \tau / \Delta x<1 / 2$, which strongly reduces the problem of trajectory truncation.

To compute the substep at time $t+(n+1) \tau, R_{I+\frac{1}{2}}^{u}$ is computed at the points $N-\left(N_{\tau}+N_{\text {buf }}\right)+n+1 / 2, \ldots, N$ by the expression (8). The components $R_{I}^{v}$ and $R_{I}^{\Phi}$ are computed at the points $N-\left(N_{\tau}+N_{\text {buf }}\right)+n+1, \ldots, N$ by (9) and (10).

For the predictor part of the scheme, that is, $k=0$ in eqs. (56)-(58), the values of $\Phi$ and $v$ are computed at the points $(I, \quad t+(n+1) \tau)$ lying in the buffer $N-$ $\left(N_{\tau}+N_{\text {buf }}\right)+n+1, \ldots, N$, from the values of the previous substep in $(I-1 / 2, t+n \tau),(I, t+n \tau)$ and $(I+1 / 2, t+n \tau)$, by the eqs. (57) and (58). The field $u$ is computed on the points $(I, t+(n+1) \tau)$ in the buffer zone $N-\left(N_{\tau}+N_{\text {buf }}\right)+n+1 / 2, \ldots, N$, on the half grid point $(I+1 / 2, t+(n+1) \tau)$, from the values at the points

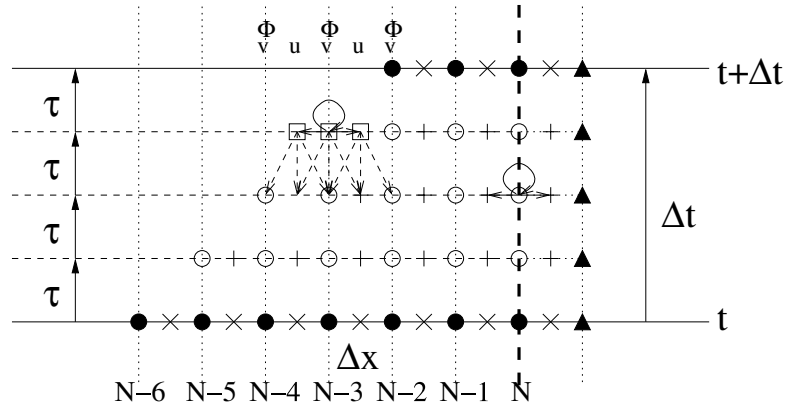

Fig. 5. The space time structure of the extrinsic predictor/corrector scheme near the right-hand boundary, with the same conventions as in Fig. 4. For the three points $(N-7 / 2, t+3 \tau)(N-3, t+3 \tau)$ and $(N-$ $5 / 2, t+3 \tau)$, indicated by the three rectangles, the space time dependence of the predictor step is given by the nine dashed arrows (three for each point). The predictor values at these three points are needed for the computation of the corrector step at point $(N-3, t+$ $3 \tau)$. The space-time dependence of the corrector is indicated by the solid arrows. The solid arrow making a loop indicates that the corrector needs the predictor value at the same space time location. The corrector step is also illustrated in $(N, t+2 \tau)$.

$(I-1, t+n \tau),(I+1 / 2, t+n \tau)$ and $(I, t+n \tau)$ by (56). This space-time dependence of the predictor step is illustrated by the dashed arrows, leading to the empty rectangles in Fig. 5.

For the corrector, that is, $k=1$, the values of $\Phi$ and $v$ are also computed at the points $(I, t+(n+1) \tau)$ lying in the same buffer $N-\left(N_{\tau}+N_{\text {buf }}\right)+n+1, \ldots, N$. However, the field $u$ is computed on the points $(I, t+(n+1) \tau)$ in the buffer zone $N-$ $\left(N_{\tau}+N_{\text {buf }}\right)+n+3 / 2, \ldots, N$. This space-time dependence of the predictor step is illustrated by the solid arrows in Fig. 5. In this way, it is not necessary to let the buffer shrink an extra point for the corrector, as was the case for the leapfrog trapezoidal scheme. And the buffer at time $t$ can be taken as $N_{\tau}+N_{\text {buf }}$.

At the boundary point $N$, we impose the characteristic boundary condition in the same way as for leapfrog trapezoidal scheme after both the predictor and the corrector step.

In the spectral model, the fields $\mathbf{X}^{-}$and $\mathbf{X}^{0}$ are present on the A grid in spectral space. They are first interpolated to the $\mathrm{C}$ grid before starting the substepping.

\section{Experimental support for extrinsic LBCs}

The aim of this section is to test the idea of externalized LBCs. All problems that could potentially originate from causes other than the extrinsic LBCs will be meticulously eliminated. Testing in a setup that resembles the behaviour of models in operational situations constitutes the next step but lies beyond the scope of this paper.

In limited-area models, the provided boundary data are taken from large-scale coupling models. Part of the inaccuracy of the LBCs is caused by the difference between the large-scale model and the coupled model, see Warner et al. (1997) for a discussion. 
The problem of the interpolation of the coupling data between provided states, with time differences that are much bigger than the model time step (of typically three or more hours, see Termonia, 2003), as occurring in real models, will be avoided by providing the coupling data at all time steps. The method in Termonia (2004) provides a technique to control the quality of the interpolation in operational models, allowing to see this a separate scientific problem.

Seven different schemes have been studied: the gridpoint version of the model with intrinsic LBCs (which will be referred to as GPINTR henceforth); the gridpoint version with the three extrinsic schemes in 3.1, 3.2 and 3.3 (GPLF, GPLFTR, GPISL, corresponding to the leapfrog, the leapfrog-trapezoidal and the iterative semi-Lagrangian respectively) and the spectral model with same three extrinsic schemes (SPLF, SPLFTR, SPISL).

Tests have also been carried out with extrinsic substepping in the spectral model on A grids. Spurious modes of $2 \Delta x$ wavelength were observed. To avoid this complication only tests with extrinsic schemes on $\mathrm{C}$ grids are presented here.

\subsection{Stability of the fast solutions}

The numerical stability of the scheme is determined by the fastest solutions which are given by

$u=F \cos \left[k\left\{x-\left(\bar{u}+c_{k}\right) t\right\}+\zeta\right]$,

$v=F \frac{f}{k c_{k}} \sin \left[k\left\{x-\left(\bar{u}+c_{k}\right) t\right\}+\zeta\right]$,

$\Phi=F \frac{1}{c_{k}} \cos \left[k\left\{x-\left(\bar{u}+c_{k}\right) t\right\}+\zeta\right]$,

with amplitude $F$, phase $\zeta$ and $c_{k}$ as defined in Section 2 . This wave corresponds to wave Courant number $\alpha_{w}=(\bar{u}+$ $\bar{c}) \Delta t / \Delta x$. Since this solution represents meteorological noise, the accuracy of the schemes to represent this solution is subordinate to the stability.

Experiments have been performed for $\Delta x=10 \mathrm{~km}, \bar{u}=$ $100 \mathrm{~m} \mathrm{~s}^{-1}$ and $f=10^{-4} \mathrm{~s}^{-1}$.

The six schemes GPINTR, GPLF, GPLFTR, GPISL, SPLFTR, SPISL turned out to be stable for any $\Delta t$, provided that the substepping time step $\tau$ was chosen to satisfy (45), (55) and (59). For time steps larger than $150 \mathrm{~s}$, the SPLF scheme became unstable by the computational mode of the leapfrog substepping. Figure 6 illustrates the tests for the schemes GPINTR, GPISL and SPISL. The scheme GPLFTR gives the same result as the the one shown for GPISL in Fig. 6b. As an illustration, the artifical extension zone between $x=1000$ and $1200 \mathrm{~km}$, where the fields are made period, is included in Fig. 6c.

Note that in the gridpoint versions in Figs. $6 \mathrm{a}$ and $6 \mathrm{~b}$, the phase error manifests itself at the right-hand boundary but not at the left-hand boundary. The net result is that the wave becomes slightly squeezed as time progresses. This does not happen in the spectral version, where the the phase shift exists everywhere in the domain. The artificial extension zone is added in Fig. 6c, showing the periodic extension.
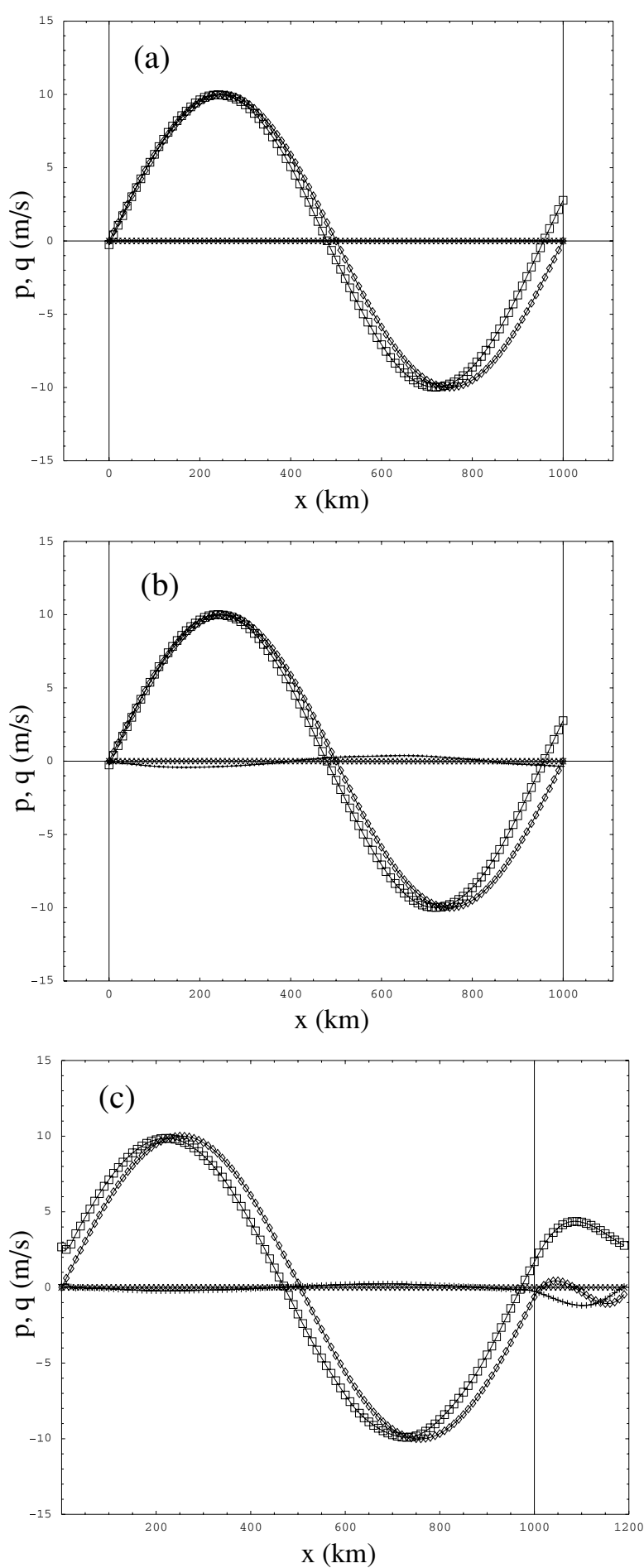

Fig. 6. Tests of the stability of the schemes for the fast solution for the schemes (a) GPINTR, (b) GPISL and (c) SPISL, for

$\Delta t=400 \mathrm{~s}, \bar{u}=100 \mathrm{~m} \mathrm{~s}^{-1}, \bar{c}=300 \mathrm{~m} \mathrm{~s}^{-1}, f=10^{-4} \mathrm{~s}^{-1}$ on a domain of length $L=1000 \mathrm{~km}$ at time $T=L /\left(\bar{u}+c_{k}\right)$, when the wave solution has travelled one wavelength through the domain. The fields shown are the characteristic values for $p(t=0)$ (diamonds, joined by solid lines), $p(T)$ (rectangles, joined by solid lines), $q(0)$ (crosses, joined by solid lines) and $q(T)$ (pluses, joined by solid line s). 
It is interesting to see that the phase error of the 2TL SISL scheme of the dynamic core creates a discrepancy between the numerical solution in the interior domain and the analytical solution. The tests in Fig. 6 show that the LBC formulation correctly handle this: they do not generate spurious reflections and the wave enters the domain even when the imposed value does not correspond to what happens at the boundary.

\subsection{Accuracy of the slow solutions}

As a meteorologically relevant test the following slow bellshaped solution

$u=0$,

$v=-2 \frac{\mathrm{e}^{\bar{\Phi}}}{f} \frac{x-x_{s}}{\sigma_{s}^{2}} \hat{\Phi} \mathrm{e}^{-\left(\frac{x-x_{s}}{\sigma_{s}}\right)^{2}}$,

$\Phi=\hat{\Phi} \mathrm{e}^{-\left(\frac{x-x_{s}}{\sigma_{s}}\right)^{2}}$.

has been used as initial condition, as in McDonald (2000). This solution is a superposition of slow solutions of the form

$u=0$,

$v=-S \frac{k}{f} \mathrm{e}^{\bar{\Phi}} \sin \left[k(x-\bar{u} t)+\zeta_{0}\right]$,

$\Phi=S \cos \left[k(x-\bar{u} t)+\zeta_{0}\right]$.

with varying wavenumber $k$ but propagating with uniform velocity $\bar{u}$.

Figure 7 shows a test of the bell-shaped solution by the schemes GPINTR, GPISL and SPISL. The result for the GPISL scheme is almost exactly the same as for the GPINTR schemedifferences were observed in the numerical output of the two model versions, but no differences can be seen in the figure.

The same test was also performed for the GPLF and GPLFTR scheme and gave exactly the same result as Fig. 7b.

As discussed in Section 2.3, for the spectral version there are the two additional sources of inaccuracy - the tiny but unavoidable leaking of the extension into the physical zone and the inaccuracy of the $\mathbf{Q}$.

As shown in the expansion (42), the accuracy deteriorates as the normalized wavenumber $\xi$ increases. It also deteriorates as the wave Courant number $\sigma$ increases, for instance when augmenting the time step $\Delta t$. Figure 8 shows some tests for different slow, balanced solutions (62) for $K=1,2,4,8$, as defined in (40) and for time steps $\Delta t=100 \mathrm{~s}$ and $400 \mathrm{~s}$, as a way to quantify both $\xi$ and $\sigma$ dependence.

Figure 9 shows the same experiment as in Fig. 7 but for the scheme SPISL and with $\bar{u}=12.5 \mathrm{~m} \mathrm{~s}^{-1}$ and $\Delta t=400 \mathrm{~m} \mathrm{~s}^{-1}$. In contrast to the artificial setups in Fig. 8, this represents a test with the same scheme but for a meteorologically relevant situation. To illustrate the sources of inaccuracy the time evolution is shown here at four times. At time $T=L / \bar{u}$ when the bell has crossed the domain, a small feature can be noticed near the left-hand boundary. We believe that this is a
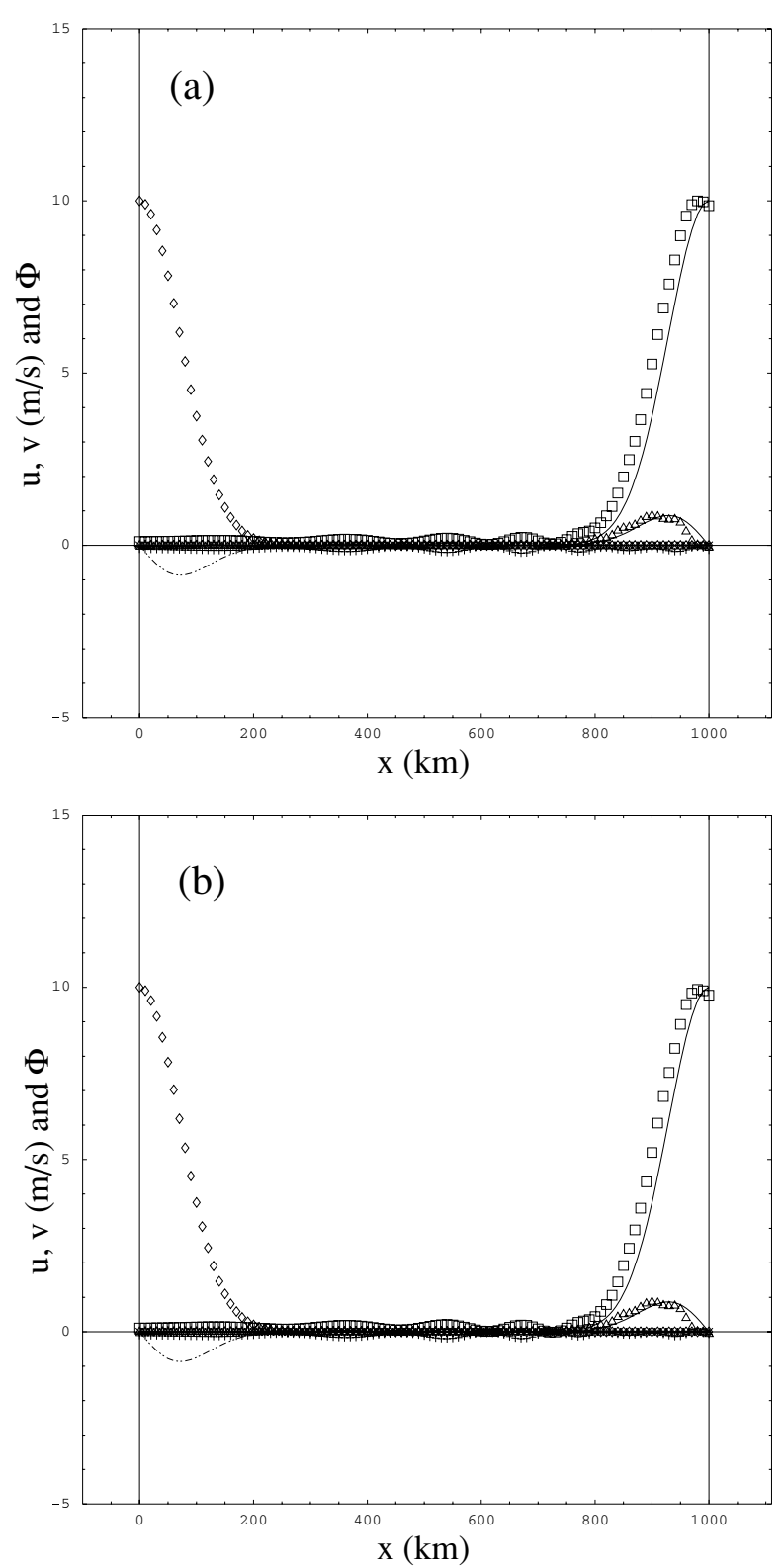

Fig. 7. Bell-shaped slow solution computed by scheme (a) GPINTR and (b) GPISL with $\Delta t=416 \mathrm{~s}$ and $\bar{u}=100 \mathrm{~m} \mathrm{~s}^{-1}$, both on a domain of length $L=1000 \mathrm{~km}$. The fields are $u(0)$ (crosses), $v(0)$ (dash dot dot), $\Phi(0)$ (diamonds), $u(T)$ (pluses), $v(T)$ (triangles) and $\Phi(T)$ (rectangles), with $T=L / \bar{u}$, the time to travel through the entire domain. The solid lines represent the analytic solution.

combination of some contamination of the extensions zone and the inaccuracy of the $\mathbf{Q}$ operator. The results for SPLFTR are similar.

Although the inaccuracy of the $\mathbf{Q}$ operator manifests itself clearly in the tests in Fig. 8, the net result stays small in the test of the meteorological situation in Fig. 9 and remains restricted to about ten grid points near the boundary. 

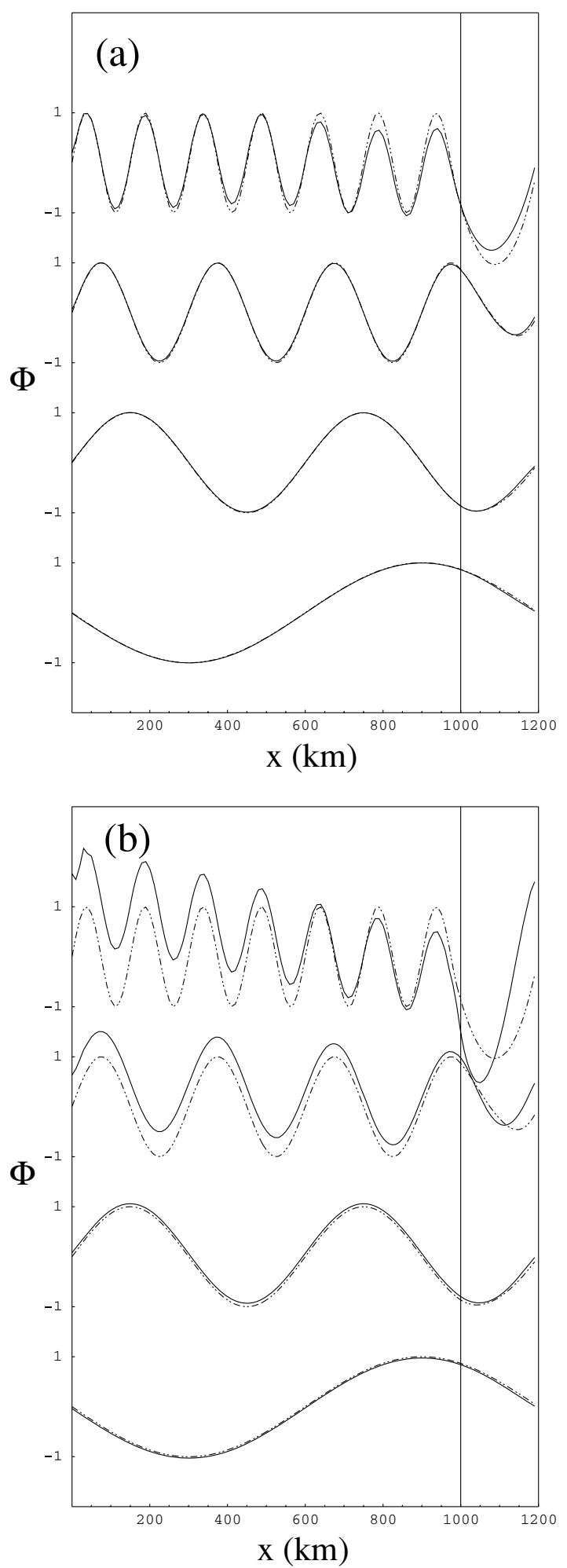

Fig. 8. Tests with the SPISL scheme (solid lines) of balanced waves (slow solutions) with wavenumbers $K=1, K=2, K=4, K=8$ (from bottom to top) for (a) $\Delta t=100 \mathrm{~s}$ and (b) $\Delta t=400 \mathrm{~s}$. The other parameters are taken $\bar{u}=12.5 \mathrm{~m} \mathrm{~s}^{-1}, \Delta x=10 \mathrm{~km}$. The true solution is also indicated (dash dot dot).

\subsection{Better treatment of the trajectories by an enhanced buffer zone}

A major reason for the reflections in Fig. 7 are the semiLagrangian trajectories of the 2TL SISL scheme and their truncations. McDonald (2000) proposes a few approaches for this scheme to address this problem.

In the schemes with the extrinsic LBCs, the combination of the externalization with substepping provides an alternative solution to this. First, if the extrinsic LBCs are computed with $N_{\text {buf }}>$ $\bar{u} \Delta t / \Delta x$, the trajectories of the points used in the interior will not lie beyond the boundary. Additionally, by construction the advection Courant number of the substepping is small enough to guarantee a high accuracy of the trajectory truncation.

Figure 10 illustrates this by two tests with GPISL and SPISL run, with $N_{\text {buf }}=5$, with exactly the same settings as in Fig. 7. This option with its corresponding improvement is impossible in the GPINTR scheme in Fig. 7a. For the GPISL case the reflections almost disappear completely. This is also the case for the spectral model SPISL, but in this case the artefact is still present near the lef-handt boundary, as well as a smaller one at the right-hand boundary.

\subsection{An adjustment and radiation test}

Some tests have also been performed for the Rossby adjustment problem as described by Gill (1982) and tested by McDonald (2000). This represents the evolution of the unbalanced initial state $u(t=0)=0, v(t=0)=0, \phi(t=0, x \leq L / 2)=10$ and $\phi(t=0, x>L / 2)=-10$. to a geostrophic steady-state. The test is entirely the same as in McDonald (2000): $\Delta x=100 \mathrm{~km}$, $\Delta t=10 \mathrm{~min}$ and $\bar{u}=1 \mathrm{~m} \mathrm{~s}^{-1}$, with a domain of length $L=$ $30000 \mathrm{~km}$, which is large compared with the Rossby number of deformation. The characteristic values are imposed as described in Section 3.

Figure 11 shows the state after $10 \mathrm{~d}$. It represents the known exact solution (given in Gill, 1982), almost identical as in McDonald (2000). The GPISL gives the same result and is therefore not given here (the plots could not be distinguished visually).

Additionally a radiation experiment was performed as in McDonald (2002) given by the initial condition,

$u=0, \quad v=0, \quad \Phi=\hat{\Phi} \mathrm{e}^{-\left(\frac{x-x_{s}}{\sigma_{s}}\right)^{2}} \sin \frac{16 \pi x}{L}$.

The tests are shown in Fig. 12 for the GPISL and SPISL scheme. The model was run on the shown domain while imposing the characteristic values $v_{0}^{h}, p_{0}^{h}$ and $q_{N}^{h}$. This state creates two wave packets propagating in the opposite directions. At $t=0.8776 \mathrm{~h}$, both have left the domain without spurious reflections, producing equivalent results to the ones obtained by McDonald (2002). 
Fig. 9. Time evolution of the bell-shaped solution with the SPISL scheme at times (a) $t=0,(\mathrm{~b}) t=L /(2 \bar{u}),(\mathrm{c}) t=L / \bar{u}$ snd (d) $t=3 L /(2 \bar{u})$, for $\Delta t=400 s$ and $\bar{u}=12.5 \mathrm{~m} \mathrm{~s}^{-1}$. The conventions are the same as in Fig. 7.
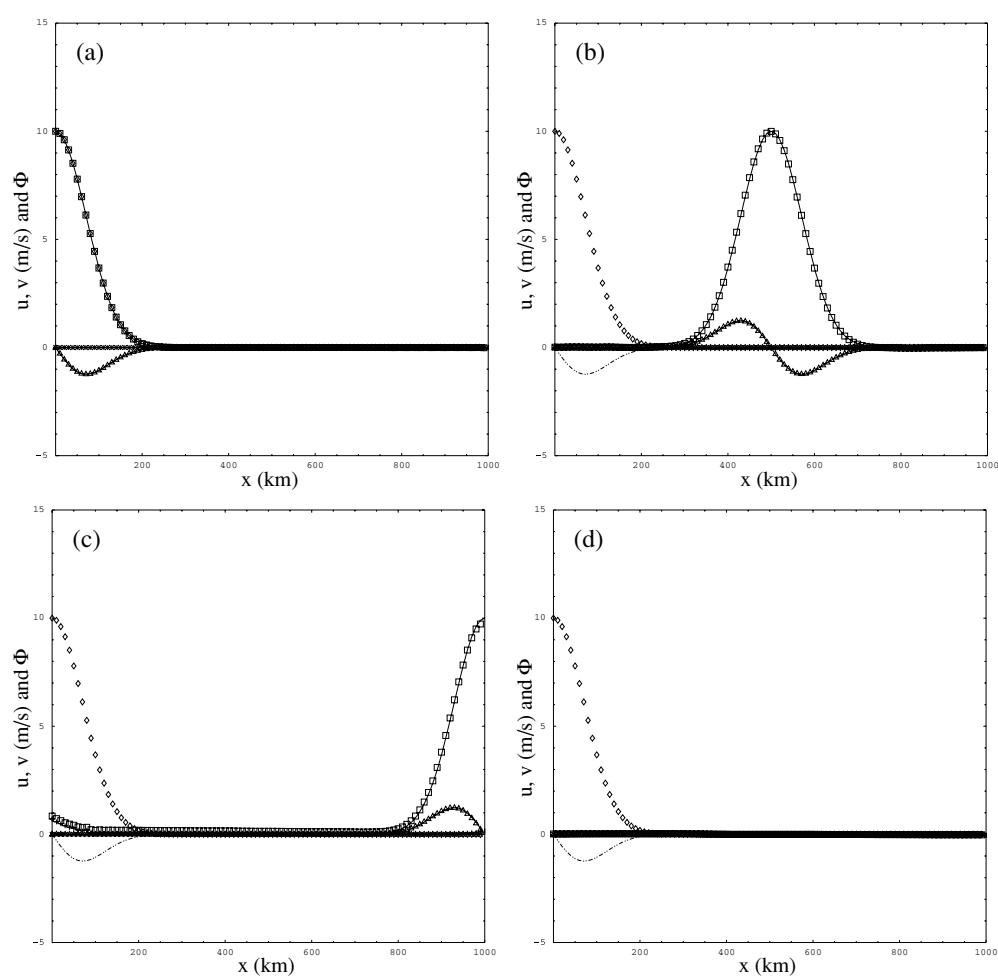

\section{Discussion and outlook}

The tests show that, at this stage, the idea of externalized extrinsic LBCs is promising. The major finding is that 'stable' LBCs are possible in a gridpoint model 'and' in a spectral model using this approach. In the gridpoint model, the same accuracy could be obtained as for the intrinsic LBC model, and the use of the enhanced buffer allows to get even higher accuracy. The issues that are still open solely pertain to the accuracy in the spectral model.

As explained and illustrated in this paper, the main advantages of extrinsic schemes are the following.

1. Although the dynamic core is semi-Lagrangian, this allows to use explicit schemes at the boundaries, which eliminates the problem of the trajectory truncations.

2. Any LBC scheme developed for a spectral model can be used for a gridpoint model and vice versa. This is illustrated in the test in Fig. 7. This also means that one can develop the dynamic core without having to consider the details of the LBC scheme.

3. For a given dynamic core, an $\mathrm{LBC}$ scheme can be replaced by another. In a 2TL SISL scheme, this means, for instance, that one can develop the numerical solver of the Helmholtz equation once and keep using it when scientific progress is made in the (externalized) formulation of the LBCs.

4. Externalization allows to circumvent the impossibility to impose LBCs in a spectral Helmholtz equation.
Of course the stability of the scheme with externalized LBCs is determined by the stability of the dynamic core and of the extrinsic scheme, specifically by the weakest of both. For instance, in the case of the leapfrog scheme, the unstable computational mode is the determining factor.

The fact that the semi-implicit operators are computed in gridpoint space and then inverted in spectral space decreases the accuracy of the method in the case of the spectral model. This a priori is not a shortcoming of the idea of the externalization but a problem of the difference of the gridpoint derivatives versus the spectral derivatives. The result is a decreased accuracy of the scheme compared with what could be obtained in the gridpoint model. In this paper, only one gridpoint version of the operator has been studied, with limited accuracy of $\mathbf{Q}$. The search for more accurate choices will be the subject of future studies.

Specifically, the weak points of the current tests in the spectral setup with the periodic extension of the ALADIN model are

1. the potential non-local contamination of the extension zone into the physical zone and

2. the inaccuracy of the $\mathbf{Q}$ operator.

This manifests itself in the artefact in a zone of about ten gridpoints near the boundary in Figs. 9 and 10, while the run in the rest of the domain remains unharmed. Mind that the Davies scheme in the ALADIN model uses an artificial zone of about eight gridpoints width near the boundary where the forecast is not to be taken as physically relevant. So, notwithstanding the artefact, the tests have produced satisfactory results. 

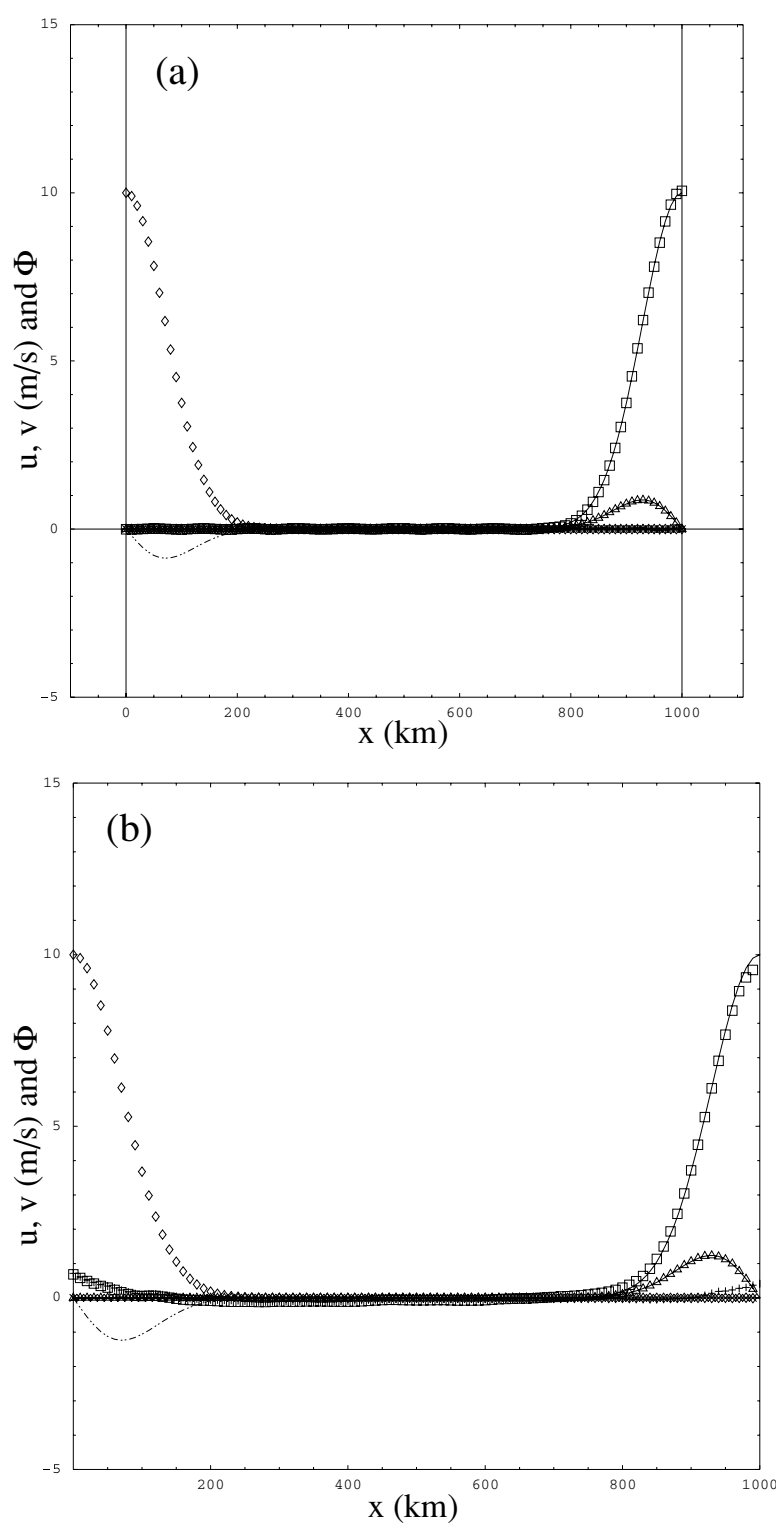

Fig. 10. Bell-shaped solution computed by scheme (a) GPISL and (b) SPISL both with $\Delta t=416 \mathrm{~s}, \bar{u}=100 \mathrm{~m} \mathrm{~s}^{-1}$ and buffering $N_{\text {buf }}=5$. The conventions are the same as in Fig. 7.

A good candidate for improving the formulation of the periodic extension in the spectral model and creating higher accuracy of the derivatives is the proposal of Boyd (2005). This may allow to use centred derivatives in (32) and (35) by the points lying outside of the domain.

The use of such operators is already being done in the ALADIN model for the current implementation of the Davies scheme, see Radnóti (1995). However, in that case, all derivatives are computed in spectral space. So, there is no problem of accuracy in the existing ALADIN model. However, the existence of a null space of these operators and potential problems thereof have never been investigated. The fact that no serious shortcom-

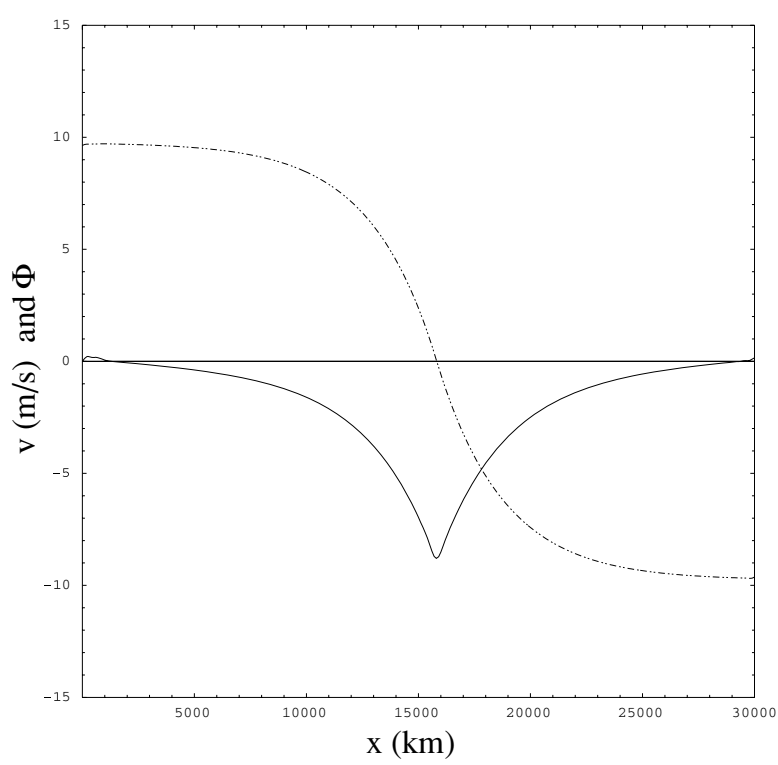

Fig. 11. Spectral model SPISL: $\Delta x=100 \mathrm{~km}, t=10 \mathrm{~d}, \Delta t=600 \mathrm{~s}$, $\bar{u}=1 \mathrm{~m} \mathrm{~s}^{-1}$ of the fields $v$ (solid) and $\Phi$ (dash dot dot). Tests were performed for GPISL and gave exactly the same fields as in this figure.

ings have been observed in the research runs and the operational suites of the ALADIN runs suggests that even if the null-spaces modes propagate, their effect remains negligibly small.

The trick of computing the semi-implicit operator in gridpoint space before going to spectral space could become useful in a broader context in spectral models, provided the null space of the $\mathbf{Q}$ operator can be understood better and kept under control. Indeed, it allows to pull any gridpoint computation of a variable (e.g. $\tilde{\mathbf{X}}_{L, R}^{+}$in Fig. 2) to the end of the time step, without substantially being distorted by the spectral part of the dynamics.

The relation between the error propagating into the domain and the error originating from the null-space modes of the $\mathbf{Q}$ operator is a complicated one. As shown in eq. (43), the nullspace modes have an exponential spatial dependence. Once they start to propagate, there is a priori no reason for them to stay exponential. This makes it difficult to diagnose this problem and to distinguish its contribution from other error sources. In fact, the error growth due to errors in the LBCs is poorly understood. The first proposal for a general formulation has been given only recently by Nicolis (2007). The approach in that paper may become useful in minimizing the error of the LBCs, by a proper choice of the the gridpoint part of the $\mathbf{Q}$ operator, although the analysis may have to be carried out in a simpler advective model than the present one.

The substepping scheme is quite demanding in computing resources due to its need of rather large buffers near the boundary. For instance for $\Delta t=400 \mathrm{~s}, \Delta x=10 \mathrm{~km}$ and $\bar{c}=300 \mathrm{~m} \mathrm{~s}^{-1}$, eq. (59) requires $N_{\tau}=25$ substeps and a buffer zone at the beginning of the substepping of $N_{\text {buf }}+25$ gridpoints. While the substepping itself would be relatively cheap in realistic 3-D 

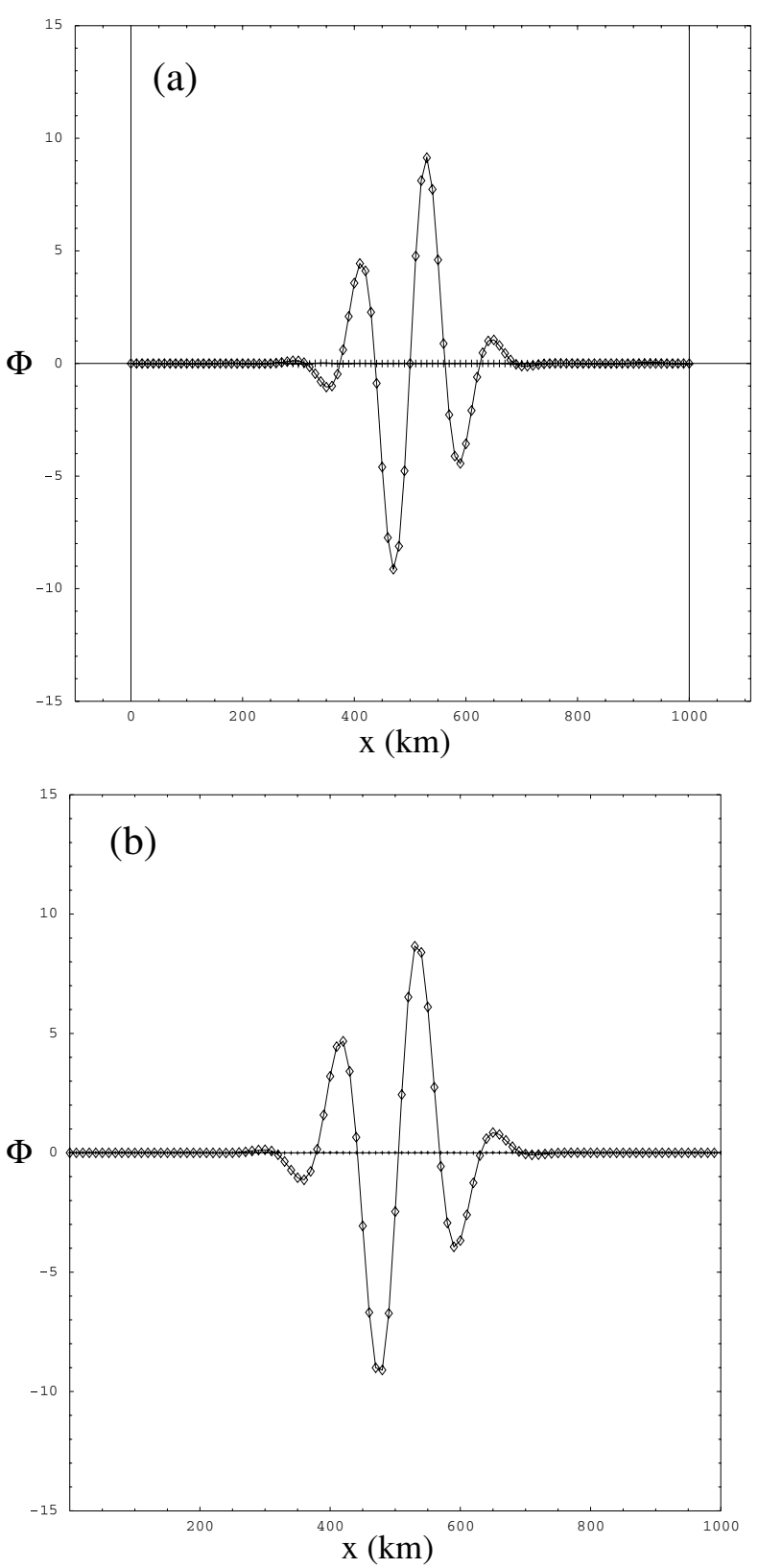

Fig. 12. Adjustment of the field $\Phi$ at time $t=0 \mathrm{~s}$ (indicated by diamonds joined by solid lines) after time $t=0.8776 \mathrm{~h}$ (pluses joined by solid lines) with $\Delta t=25 \mathrm{~s}$ and $\bar{u}=0.0001 \mathrm{~m} \mathrm{~s}^{-1}$, for the (a) GPISL and (b) SPISL.

model codes, it may lead to a substantial increase in the communication in parallel model codes.

Alternative extrinsic schemes should be investigated to reduce this computational cost, ideally semi-implicit schemes. To implement a semi-implicit scheme, it is necessary to impose some conditions at the interior of the buffer zone, that is, at the points $N-\left(N_{\tau}+N_{\text {buf }}\right)+n$ for the shrinking right-hand buffer zone for substep $(n+1) \tau$. With the use of interior boundary condi- tions the computing cost would be reduced for two reasons: (1) a smaller buffer zone and (2) larger time steps hopefully eliminating the need to rely on substepping. However, this has not been studied in this paper since this would have complicated the present study by making it more difficult to distinguish the difficulties originating from this compromise from the difficulties due to the externalization idea itself. The present schemes can serve as a reference for developing alternatives.

In contrast, using a buffer zone together with substepping allows to solve the problem of the trajectory truncation, as shown in Fig. 10. This problem is difficult to solve in an intrinsic LBC formulation with a semi-Lagrangian scheme.

Put differently, the value of the choice for substepping in the present study lies primarily in its usefulness to follow an exhaustive testing procedure of extrinsic LBCs (everything can be controlled by the substepping). Moreover, the substepping schemes are very different from the 2TL SISL in the interior domain making the test of the hypothesis even stronger.

Within the present time step organization of the type used in the ALADIN model (see the description of the AAA models by Termonia and Hamdi, 2007), it would not be necessary to compute the physics in the extrinsic LBC scheme. In this model, the physics is computed and coupled to the dynamics before the explicit part of the dynamics. So, in Fig. 2, it can be coupled to $\mathbf{X}^{-}$and $\mathbf{X}^{0}$ before they are provided to the extrinsic LBC scheme (as in indicated by the left-hand side double-lined arrow). In the case of semi-Lagrangian averaging of the physics-dynamics coupling, as is, for instance, done in the SLAVEPP scheme of the IFS model proposed by Wedi (1999), it should be considered not to average the values that are input to the extrinsic scheme.

The biggest problems discussed in this paper were related to the inversion of the implicit operator and the truncation of the semi-Lagrangian trajectories. These problems are not present in explicit and/or Eulerian codes. Since this study was motivated by a need for alternative LBCs in the ALADIN model, we started with the most difficult case. We believe that, even if it presently seems less interesting, externalizing the LBCs is technically more likely to work in Eulerian and explicit dynamic cores.

\section{Acknowledgments}

This work benefited from useful discussions with Aidan McDonald and Pierre Bénard. We very much appreciate the numerous helpful comments of Pierre Bénard and Jean-François Geleyn on the manuscript. The manuscript was also substantially improved by numerous comments of one of the anonymous reviewers.

\section{References}

ALADIN International team, 1997. The ALADIN project: mesoscale modelling seen as basic tool for weather forecasting and atmospheric research. World Meteorol. Organ. Bull. 46, 317-324. 
Arakawa, A. and Lamb, V. R. 1977. Computational design of the basic dynamical processes of the UCLA general circulation model. Meth. Comput. Phys. 17, 173-265.

Best, M. J., Beljaars, A., Polcher, J. and Viterbo, P. 2004. A Proposed Structure for Coupling Tiled Surfaces with the Planetary Boundary Layer. J. Hydrometeor. 5, 1271-1278.

Bénard, P. 2003. Stability of Semi-Implicit and Iterative CenteredImplicit Time Discretizations for Various Equation Systems Used in NWP. Mon. Wea. Rev. 131, 2479-2491.

Boyd, J. P. 2005. Limited-Area Fourier Spectral Models and Data Analysis Schemes: Windows, Fourier Extension, Davies Relaxation, and All That. Mon. Wea. Rev. 133, 2030-2042.

Davies, H. C. 1976. A lateral boundary formulation for multilevel prediction models. Q. J. Roy. Meteorol. Soc. 102, 405-418.

Durran, D. R. 1999. Numerical Methods for Wave Equations in Geophysical Fluid Dynamics, Springer-Verlag, New York, 465 pp.

Gill, A. E. 1982. Atmosphere-Ocean Dynamics, Academic Press, CA, $662 \mathrm{pp}$.

Haugen, J. E. and Machenhauer, B. 1993. A Spectral Limited-Area Model Formulation with Time-Dependent Boundary Conditions Applied to the Shallow-Water Equations. Mon. Wea. Rev. 121, 26182630.

McDonald, A. 2000. Boundary Conditions for Semi-Lagrangian Schemes: Testing some Alternatives in One-Dimensional Models. Mon. Wea. Rev. 128, 4084-4096.

McDonald, A. 2002. Testing Transparent Boundary Conditions for the Shallow Water Equations in a Nested Environment. HIRLAM technical report 54, (Available from A. McDonald, Irish Meteorological Service, Glasnevin Hill, Dublin 9, Ireland) 28 pp.

McDonald, A. 2003. Transparent Boundary Conditions for the ShallowWater Equations: Testing in a Nested Environment. Mon. Wea. Rev. 131, 698-705.

McDonald, A. 2005. Transparent lateral boundary conditions for baroclinic waves: a study of two elementary systems of equations. Tellus 57A, 171-182.

McDonald, A. 2006. Transparent lateral boundary conditions for baroclinic waves II. Introducing potential vorticity waves. Tellus $\mathbf{5 8 A}$, 210 220.
Nicolis, C. 2007. Dynamics of Model Error: The Role of the Boundary Conditions. J. Atmos. Sci. 64, 204-215.

Oliger, J. and Sundström, A. 1978. Theoretical and practical aspects of some initial boundary value problems in fluid dynamics. SIAM J. Appl. Math. 35, 419-446.

Radnóti, G. 1995. Comments on "A Spectral Limited-Area Formulation with Time-Dependent Boundary Conditions for the Shallow-Water Equations". Mon. Wea. Rev. 123, 3122-3123.

Robert, A. J. 1966. The integration of a low order spectral form of the primitive meteorological equations. J. Meteorol. Soc. Japan 44, 237244.

Robert, A. J. 1969. The integration of a spectral model of the atmosphere by the implicit method. In: Proceedings of the WMO/IUGG Symposium on NWP. Japan Meteor. Agency, Tokyo, VII $19-24$.

Robert, A. J. 1981. A stable numerical integration scheme for the primitive meteorological equations. Atmos. Ocean 19, 35-46.

Sundström, A. and Elvius, T. 1979. Computational problems related to limited area modelling. In: Numerical Methods Used in Atmospheric Models, Volume II of GARP Publication Series No. 17, World Meteorological Organisation, Geneva, 379-416.

Termonia, P. 2003. Monitoring and Improving the Temporal Interpolation of Lateral-Boundary Coupling Data for Limited-Area Models. Mon. Wea. Rev. 131, 2450-2463.

Termonia, P. 2004. Monitoring the Coupling-Update Frequency of a Limited-Area Model by Means of a Recursive Digital Filter. Mon. Wea. Rev. 132, 2130-2141.

Termonia, P. and Hamdi, R. 2007. Stability and accuracy of the physicsdynamics coupling in spectral models. Q. J. R. Meteorol. Soc. 133, 1589-1604.

Warner, T. T., Peterson, R. A. and Treadon, R. E. 1997. A Tutorial on Lateral Boundary Conditions as a Basic and Potentially Serious Limitation to Regional Weather Forecasting. Bull. Am. Meteorol. Soc. 78, 2599-2617.

Wedi, N. P. 1999. The numerical coupling of the physical parameterizations to the "dynamical" equations in a forecast model. ECMWF Tech. Memo. 274, European Centre for Medium-Range Weather Forecasts. ECMWF, Reading, United Kingdom. 